\title{
Radiation-Balanced Lasers: History, Status, Potential
}

\section{Galina Nemova}

Citation: Nemova, G. RadiationBalanced Lasers: History, Status, Potential. Appl. Sci. 2021, 11, 7539. https://doi.org/10.3390/app11167539

Academic Editors: David Andrews and Joachim Hein

Received: 20 June 2021

Accepted: 13 August 2021

Published: 17 August 2021

Publisher's Note: MDPI stays neutral with regard to jurisdictional claims in published maps and institutional affiliations.

Copyright: (C) 2021 by the author. Licensee MDPI, Basel, Switzerland. This article is an open access article distributed under the terms and conditions of the Creative Commons Attribution (CC BY) license (https:// creativecommons.org/licenses/by/ $4.0 /)$.
Department of Electrical Engineering, Polytechnique Montréal, 2500 ch. de Polytechnique, Montréal, QC H3T 1H4, Canada; galina.nemova@videotron.ca

\begin{abstract}
The review of history and progress on radiation-balanced (athermal) lasers is presented with a special focus on rare earth (RE)-doped lasers. In the majority of lasers, heat generated inside the laser medium is an unavoidable product of the lasing process. Radiation-balanced lasers can provide lasing without detrimental heating of laser medium. This new approach to the design of optically pumped RE-doped solid-state lasers is provided by balancing the spontaneous and stimulated emission within the laser medium. It is based on the principle of anti-Stokes fluorescence cooling of RE-doped low-phonon solids. The theoretical description of the operation of radiationbalanced lasers based on the set of coupled rate equations is presented and discussed. It is shown that, for athermal operation, the value of the pump wavelength of the laser must exceed the value of the mean fluorescence wavelength of the RE laser active ions doped in the laser medium. The improved purity of host crystals and better control of the transverse intensity profile will result in improved performance of the radiation-balanced laser. Recent experimental achievements in the development of radiation-balanced RE-doped bulk lasers, fibre lasers, disk lasers, and microlasers are reviewed and discussed.
\end{abstract}

Keywords: radiation-balanced lasers; athermal lasers; solid-state lasers; laser cooling of solids; rare earth-doped materials

\section{Introduction}

The replacement of flash lamps by laser-diode pumping for solid-state lasers has brought a very important breakthrough in the laser technology, in particular for highpower lasers [1,2]. Compared to flash-lamp pumps, laser diodes have led to a significant benefit in efficiency, simplicity, compactness, reliability, and cost. At the same time the thermal problem has come into existence for high power lasers. Special care concerning thermal management is necessary to develop efficient high-power lasers.

To solve the thermal problem, the classical rod solid-state laser medium design has been replaced by new approaches including fibre lasers, slab lasers, and thin-disk lasers. The very high surface-to-volume ratio and optical guidance have provided tremendous progress in the power scalability of high-power lasers.

The benefit of using a rare earth (RE)-doped single-mode optical fibre as a laser medium was realized by Snitzer in 1961 [3]. In this paper, the use of fibres as dielectric waveguides to provide a resonant structure for an optical maser operating in the $0.6 \mu \mathrm{m}$ wavelength region was proposed. In the mid-1980s, research of single-mode optical fibre lasers was carried out very intensively [4-7]. A decade later, fibre structures with large mode areas based on multimode optical fibres were proposed to solve the problem [8]. The schemes, in which the amplification takes place in an RE-doped fibre cladding, have been proposed as a solution of the problem as well [9]. Unfortunately, even these schemes are limited by thermal lensing if a high-quality beam is required. Water cooling is not suitable for fibres with limited chemical stability, such as fluoride fibres. Lowering of the dopant concentration and increasing the length of the fibre makes cooling easier, but increases the chance of nonlinear effects including stimulated Brillouin scattering (SBS) 
and stimulated Raman scattering (SRS), which result in depletion of the amplified signal in ion-doped lasers.

In 1972, Martin and Chernock [10] proposed a new approach to the development of solid-state lasers. It is based on the idea of propagating laser beams in a direction that averages the temperature gradients in the gain medium [11]. The laser beam is injected into the slab so that it will allow the beam to make multiple total internal reflections from the polished sides as it propagates down the slab.

In 1991, Giesen et al. proposed to power a scale $\mathrm{Yb}^{3+}:$ YAG laser using a thin-disk laser design. The first thin-disk laser was demonstrated in 1994 [12,13]. The core concept of the thin-disk lasers is the use of a thin, disk-shaped active medium that is cooled through one of the flat faces of the disk. The cooled face is used as a mirror of the resonator. This face cooling minimizes the transversal temperature gradient, as well as the phase distortion transversal to the direction of the beam propagation, providing a laser beam of outstanding quality.

In 1999, Bowman [14] proposed a radiation-balanced (athermal) RE-doped bulk laser, which operates without internal heating. In this laser, all photons generated in the laser cycle are annihilated with the cooling cycle, that is, the heat generated from stimulated emission is offset by cooling from anti-Stokes emission. Athermal lasers, which are free from all thermal effects, provide a tremendous potential for an increase in the output power, maintaining a high-quality output laser beam. Beginning in 1999, several kinds of radiationbalanced lasers, including fibre lasers, disk lasers, and microlasers, have been proposed and developed. Some of these lasers have been discussed in [15], while others are considered in this review. Thermal lensing arising in traditional lasers from the quantum-defect heating is briefly discussed in Section 2. Section 3 is devoted to the theory of radiation-balanced lasing. Recent achievements in the development of radiation-balanced RE-doped bulk lasers, fibre lasers, disk lasers, and microlasers are presented in Section 4.

\section{Laser Heat Management}

Most materials are not optically active. They simply convert absorbed laser power directly into heat. If $\alpha_{a}$ is an absorption coefficient describing the absorption process and $I_{L}$ is the laser intensity, the absorbed power density is equal to $\alpha_{a} I_{L}$. This is not the case for laser media, which are optically active. The laser performance is based on efficient reemitting of absorbed pump light. The amount of heat generated in a laser depends on the pump power and the emission spectra.

It is well known that population inversion is a necessary condition for lasing. It is impossible to reach the population inversion in a two-level system. Three or four level schemes are required. As a result, the reradiated light is normally red-shifted relative to the pump (Figure 1).

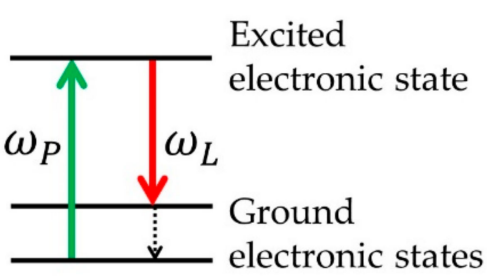

(a)

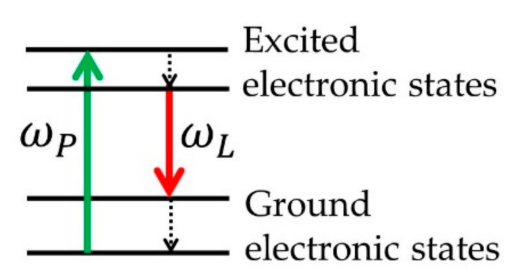

(b)

Figure 1. Energy levels and relevant excitation and decay processes for (a) three-level and (b) fourlevel lasers.

By this means, every emitted laser photon contributes a fraction of its energy,

$$
\eta_{Q D}=1-\omega_{L} / \omega_{P}
$$

to heating the laser material through phonon generation. Here $\eta_{Q D}$ is the quantum defect, $\omega_{P}$ and $\omega_{L}$ are the frequencies of the pump and laser photons, respectively. 
A study of thermal effects in a crystal requires the calculation of the temperature field at each point of the crystal. This can be achieved with the heat equation. For analytical solutions to the heat equation to be obtained, some assumptions must be imposed on the laser system. Let us suppose that the pump profile is axisymmetric, and the thermal conductivity of the laser medium does not depend on temperature and can be considered as a constant. In this case, the heat equation has the form [16]:

$$
\frac{1}{r} \frac{\partial}{\partial r}\left(r \frac{\partial T}{\partial r}\right)+\frac{\partial^{2} T}{\partial r^{2}}=-\frac{Q}{\mathrm{~K}_{c}}
$$

where $T$ is the temperature, $r$ is the radial coordinate of a point inside the laser medium (see insert in Figure 2), $K_{c}$ is the thermal conductivity, and $Q$ is the thermal power.

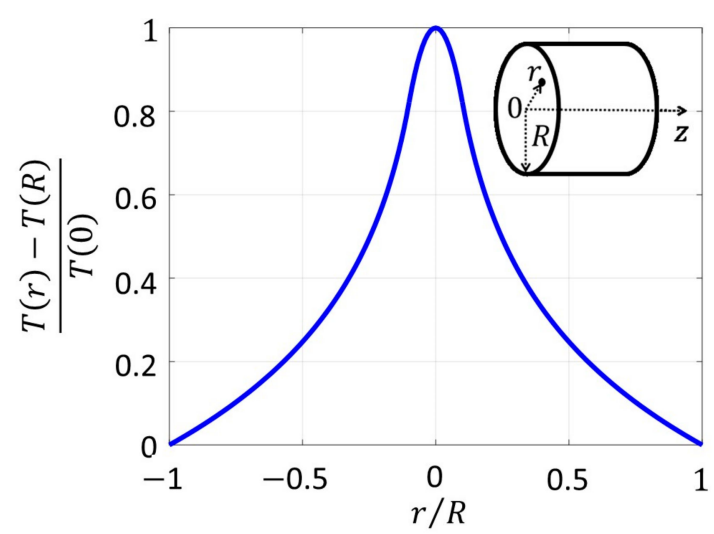

Figure 2. Normalized temperature distribution in a plane perpendicular to the $z$-axis for a laser medium pumped by a top-hat-profile laser beam. Inset is the laser medium with the radius $R$.

In a vast majority of laser schemes, the pump beam profile can be described by a superGaussian function. The general solution of the heat Equation (2) for a super-Gaussian beam of any order, as well as for Gaussian and top-hat pump beams, can be found in [17-20]. As one can see in these solutions, heat generated in the laser's interior produces a temperature rise in the center of the laser medium relative to its edge. As an example, normalized temperature distribution for a laser crystal pumped by a top-hat-profile pump beam is presented in Figure 2.

The temperature rise in the laser medium produces several undesirable effects. It reduces the lifetime of the laser active ions and increases losses. The temperature gradient causes the strain distribution inside the laser medium. The strain-induced modification of the refractive index results in depolarization and degradation in beam quality. It limits the average laser output power [21]. All these effects are known as thermal lensing. They are particularly important in the case of high-power lasers. The very high temperature rise can even melt or burn the laser medium (thermal damage). In most laser structures, beam quality degradation limits laser power below thermal damage.

As mentioned earlier, the problem of laser power scaling can be solved by reducing or eliminating the heat generated during the optical pumping and lasing process using optical cooling by anti-Stokes fluorescence within the laser medium to balance the heat generated by the Stokes shifted stimulated emission. The theory of this approach is considered in the next section.

\section{Theory of Radiation-Balanced Lasing}

In this part of the paper, I develop a model permitting to investigate the fundamental limitations of laser thermal performances. Let us consider a quasi-three-level material in which radiative losses dominate at room temperature emission and absorption spectra overlap near the lasing wavelength. This requirement is a critical material characteristic for 
a potential radiation-balanced laser. Indeed, there must be a sufficiently strong absorption in the spectral region near the lasing wavelength to allow effective coupling of the pump.

As one can see in Equation (1) as the frequency of the pump, $\omega_{P}$, approaches the frequency of the laser signal, $\omega_{L}$, the quantum defect reduces, and the heat power generated by spontaneous emission reduces, too. A minimum value for the laser quantum defect can be estimated as $k_{B} T / \hbar \omega_{P}$. It corresponds to the least amount of heat power generated by the stimulated emission providing laser operation. Let us take into account spontaneous fluorescence. Its power cannot be neglected unless the average stimulated emission rate far exceeds the rate of spontaneous emission. Spontaneous fluorescence of the laser medium can contribute significantly to radiation power flow. In certain situations, it can lead to cooling which can compensate for the laser induced heating.

\subsection{Pringsheim's Cooling}

The idea to remove the thermal energy from a system optically using anti-Stokes fluorescence was first proposed by Pringsheim in 1929 [22]. In 1995, laser cooling with anti-Stokes fluorescence was demonstrated for RE-doped solids [23]. In this first proof-ofprinciple experiment, a high-purity ytterbium $\left(\mathrm{Yb}^{3+}\right)$-doped fluorozirconate $\mathrm{ZrF}_{4}-\mathrm{BaF}_{2}-$ $\mathrm{LaF}_{3}-\mathrm{AlF}_{3}-\mathrm{NaF}-\mathrm{PbF}_{2}$ (ZBLANP) glass sample was cooled down to only $0.3 \mathrm{~K}$ below room temperature.

Each energy level of the RE ions doped into the crystal or glass host splits into a set of sublevels as a result of the Stark effect. These sets of sublevels are known as Stark level manifolds. In order to realize cooling with anti-Stokes fluorescence in an RE-doped sample, electrons must be excited from the top of the ground manifold to the bottom of the excited manifold of the RE ions. This implies that the pump wavelength, $\lambda_{P}=2 \pi c / \omega_{P}$, must be in the long wavelength tail of the absorption spectrum. After thermalization accompanied by phonon absorption, anti-Stokes fluorescence photons remove energy from the system (Figure 3).

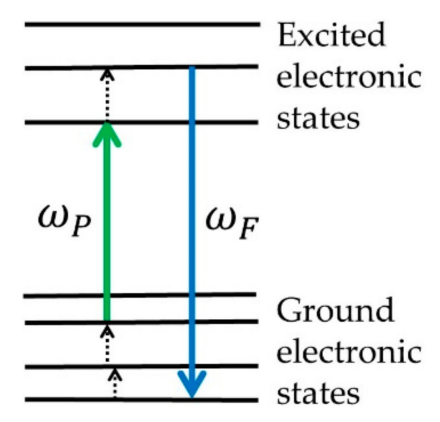

Figure 3. Energy levels and relevant excitation and decay processes for the laser-cooled RE-doped sample. $\omega_{P}$ and $\omega_{F}$ are the pump and the mean fluorescence frequencies, respectively.

The efficiency of laser cooling can be estimated as a difference between the energy of the mean fluorescence photon, $\hbar \omega_{F}$, and the energy of the pump photon, $\hbar \omega_{P}$, normalized by the energy of the pump photon [24]:

$$
\eta_{\text {cool }}=\frac{\hbar \omega_{F}-\hbar \omega_{P}}{\hbar \omega_{P}}=\frac{\lambda_{P}}{\lambda_{F}}-1,
$$

where $\omega_{P}$ and $\lambda_{P}$ are the frequency and the wavelength of the pump photon, respectively.

$$
\lambda_{F}=\frac{2 \pi c}{\omega_{F}}=\frac{\int \lambda I_{F}(\lambda) d \lambda}{\int I_{F}(\lambda) d \lambda}
$$

where $\lambda_{F}$ and $\omega_{F}$ are the mean fluorescence wavelength and the mean fluorescence frequency, respectively. $I_{F}(\lambda)$ is the fluorescence intensity at the wavelength $\lambda$. 


\subsection{Athermal Lasing}

As already mentioned, in 1999, Bowman [14] suggested to use cooling with anti-Stokes fluorescence to suppress heat generated in RE-doped solid-state lasers. He developed a new solid-state bulk laser design without internal heat generation called a radiation-balanced or athermal laser. The processes of lasing and anti-Stokes cooling in athermal lasers occur in the same system of RE ions. In 2001, Andrianov and Samartsev proposed a scheme in which lasing occurs in one system of ions $\left(\mathrm{Nd}^{3+}\right)$, while anti-Stokes cooling takes place in another system of ions $\left(\mathrm{Yb}^{3+}\right)$ doped in the $\mathrm{KY}_{3} \mathrm{~F}_{10}$ laser host [25].

\subsubsection{Athermal Lasing in Ideal Systems}

Let us consider the basic concepts of a radiation-balanced (athermal) laser. A solidstate laser of this type can be often referred to as a quasi-three-level laser. The population of each sublevel within a manifold is described by Boltzmann occupation factors. We assume that transitions between these sublevels are purely nonradiative transitions, provided by phonon absorption and emission. This process takes place on a picosecond timescale and is known as thermalization. Let us consider an isotropic laser medium with the total density of the active ions $N_{T}$. Optical transitions can occur between the ground manifold and the first excited manifolds, giving rise to overlapping, thermally broadened absorption and emission spectra. These spectra are characterized by absorption, $\sigma_{a}(\lambda, T)$, and emission, $\sigma_{e}(\lambda, T)$, cross sections, respectively. They depend on a wavelength, $\lambda$, and a temperature, $T$. For radiation-balanced operation of a laser, the mean fluorescence frequency and the pump and laser frequencies must satisfy the following relation: $\omega_{F}>\omega_{P}>\omega_{L}$ (Figure 4). In this case, the system includes two cycles: the laser cycle and the cooling cycle. The laser cycle includes the pump and laser photons at the frequencies $\omega_{P}$ and $\omega_{L}$, respectively. It is accompanied by phonon generation. The cooling cycle can be considered as a cycle including the pump photon and the mean fluorescence photon at the frequencies $\omega_{P}$ and $\omega_{F}$, respectively. It is accompanied by phonon absorption.

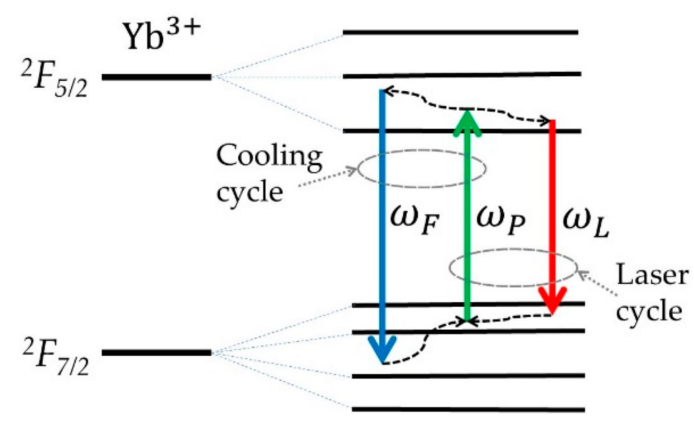

Figure 4. Energy diagram of an $\mathrm{Yb}^{3+}$-doped radiation-balanced laser. $\omega_{P}$ and $\omega_{L}$ are the pump and laser frequencies, respectively. $\omega_{F}$ is the mean fluorescence frequency.

We assume that a bandgap between the excited and ground manifolds is large compared to the energies of the phonons. As a result, the transitions between the excited and ground manifolds are purely radiative. We also assume the absence of excited-state absorption, energy transfer, radiative trapping, and background absorption. In this case, the rate equation takes the form:

$$
\begin{gathered}
\frac{d N_{2}}{d t}=W_{P}-W_{L}-\frac{N_{2}}{\tau_{R}}, \\
N_{1}+N_{2}=N_{T},
\end{gathered}
$$


where $N_{1}$ is the population of the ground manifold and $N_{2}$ is the population of the excited manifold. The last term in Equation (5) describes the total spontaneous decay rate of the upper manifold. $W_{P}$ is a pump rate, and $W_{L}$ is the stimulated emission rate:

$$
\begin{aligned}
& W_{P}=\frac{I_{P}}{\hbar \omega_{P}}\left[N_{T} \sigma_{a}^{P}-N_{2}\left(\sigma_{a}^{P}+\sigma_{e}^{P}\right)\right], \\
& W_{L}=\frac{I_{L}}{\hbar \omega_{L}}\left[N_{2}\left(\sigma_{a}^{L}+\sigma_{e}^{L}\right)-N_{T} \sigma_{a}^{L}\right] .
\end{aligned}
$$

Here $I_{P}$ and $I_{L}$ are the intensities of the pump beam and the laser signal, respectively. $\sigma_{a, e}^{P}$ and $\sigma_{a, e}^{L}$ are the absorption $(a)$ and emission $(e)$ cross sections at the pump $(P)$ wavelength, $\lambda_{P}$, and at the laser $(L)$ wavelength, $\lambda_{L}$, respectively.

Following the law of conservation of energy, one can estimate heat generated in the laser medium. Indeed, the difference between the absorbed power density and the emitted power density must be equal to the local generated heat power density:

$$
P_{\text {heat }}=\hbar \omega_{P} W_{P}-\hbar \omega_{L} W_{L}-\hbar \omega_{F} \frac{N_{2}}{\tau_{R}}
$$

Substituting Equations (5)-(8) into Equation (9), one can calculate the heat power density generated at any point of the laser medium. Equation (9) for heat power density includes both the pump, $I_{P}$, and laser, $I_{L}$, intensities in the laser medium.

If the laser system is in a steady state, $d N_{2} / d t=0$, Equation (5) takes the form

$$
W_{P}=W_{L}+\frac{N_{2}}{\tau_{R}}
$$

Let us find the relation between the pump and laser beam intensities $I_{P}$ and $I_{L}$ providing athermal operation of the laser, $P_{\text {heat }}=0$. In this case, Equation (9) becomes

$$
\hbar \omega_{P} W_{P}=\hbar \omega_{L} W_{L}+\hbar \omega_{F} \frac{N_{2}}{\tau_{R}}
$$

The change in the laser signal intensity along the length of the laser medium can be described by the well-known equation

$$
\frac{d I_{L}}{d z}=\left[\left(\sigma_{a}^{L}+\sigma_{e}^{L}\right) N_{2}-\sigma_{a}^{L} N_{T}\right] I_{L}
$$

where $z$ is the coordinate along the length of the laser medium (see Insert in Figure 2). Substituting Equations (7)-(11) into Equation (12), one can obtain the equation, which describes the laser signal at any point, $z$, along the length of the laser medium

$$
\frac{i_{L}(0)}{i_{L}(z)} \exp \left(i_{L}(z)-i_{L}(0)\right)=\exp \left(\sigma_{a}^{L} N_{T} z\right)
$$

where $i_{L}(z)=I_{L}(z) / I_{L}^{s a t}$, and $I_{L}^{s a t}=\frac{\hbar \omega_{L}}{\tau\left(\sigma_{a}^{L}+\sigma_{e}^{L}\right)}\left(\frac{\omega_{F}-\omega_{P}}{\omega_{P}-\omega_{L}}\right)$ is the saturation intensity of the laser signal. To keep the radiation balance at each point in the laser medium, the pump intensity must be distributed properly along the length of the laser medium following the relation obtained from Equations (10) and (11)

$$
i_{P}(z)=\frac{\sigma_{a}^{L}\left(\sigma_{a}^{P}+\sigma_{e}^{P}\right) i_{L}(z)}{\left(\sigma_{a}^{P} \sigma_{e}^{L}-\sigma_{a}^{L} \sigma_{e}^{P}\right) i_{L}(z)-\sigma_{a}^{P}\left(\sigma_{a}^{L}+\sigma_{e}^{L}\right)},
$$

where $i_{P}(z)=I_{P}(z) / I_{P}^{\text {sat }}$, and $I_{P}^{s a t}=\frac{\hbar \omega_{P}}{\tau\left(\sigma_{a}^{P}+\sigma_{e}^{P}\right)}\left(\frac{\omega_{F}-\omega_{L}}{\omega_{P}-\omega_{L}}\right)$ is the saturation intensity of the pump signal. As one can see in Equation (14), athermal laser operation requires careful control of the pump intensity distribution along the laser medium. Any deviation from 
this distribution will result to heating or cooling in some parts of the laser medium, which can be estimated with Equation (9). Since $i_{P}(z)>0$, one can see in Equation (14) that there is a minimum value of laser signal intensity in the laser cavity that can undergo athermal amplification:

$$
I_{L}^{\min }=\frac{\hbar \omega_{l} \sigma_{p}^{a}}{\tau_{R}\left(\sigma_{a}^{P} \sigma_{e}^{L}-\sigma_{a}^{L} \sigma_{e}^{P}\right)}\left(\frac{\omega_{F}-\omega_{P}}{\omega_{P}-\omega_{L}}\right)
$$

A comprehensive theory of the radiation-balanced (athermal) RE-doped bulk solidstate laser was developed in [14]. In [26], it was enhanced for the radiation-balanced (athermal) RE-doped fibre amplifiers.

\subsubsection{Athermal Lasing in Real Systems}

In the above section, perfect laser materials were considered, which are free from background absorption and are optically thin. Let us consider more realistic laser systems taking into account quenching, radiative trapping, and background absorption. These effects are present in all laser systems. Quenching of the laser excitation could occur through electron-phonon deactivation and through ion-ion energy transfer [27]. As a result, the radiative lifetime of an $R E$ ion, $\tau_{R}$, must be replaced by a fluorescence lifetime, $\tau_{F}$. Energy transfer losses can be arranged with a characteristic quenching lifetime, $\tau_{Q}$ [27]. Radiation trapping occurs as a result of fluorescence reabsorption and its reflection from the sample boundaries. It can complicate the modeling of the laser systems. Radiation trapping can be taken into account with the effective values for mean fluorescence wavelength, $\widetilde{\lambda}_{F}$, and lifetimes $\widetilde{\tau}_{F}, \widetilde{\tau}_{Q}$. These values can be obtained experimentally. For example, for an optically thin $\mathrm{Yb}^{3+}$ :KGW sample with the $\mathrm{Yb}^{3+}$ ion concentration $N_{T}=2.2 \times 10^{20} \mathrm{~cm}^{-3}$ the mean fluorescence wavelength and the fluorescence lifetime are $\lambda_{F}=997 \mathrm{~nm}$ and $\tau_{F}=0.275 \mathrm{~ms}$, respectively. For the $2 \mathrm{~mm} \times 8 \mathrm{~mm}, \mathrm{Yb}^{3+}: \mathrm{KGW}$ sample fluorescent trapping increases these values to $\widetilde{\lambda}_{F}=1011 \mathrm{~nm}$ and $\widetilde{\tau}_{F}=0.75 \mathrm{~ms}$. The rate equation for the radiation trapped system has the form

$$
\frac{d N_{2}}{d t}=\frac{I_{P}}{\hbar \omega_{P}}\left(N_{T} \sigma_{a}^{P}-N_{2}\left(\sigma_{a}^{P}+\sigma_{e}^{P}\right)\right)+\frac{I_{L}}{\hbar \omega_{L}}\left(N_{T} \sigma_{a}^{L}-N_{2}\left(\sigma_{a}^{L}+\sigma_{e}^{L}\right)\right)-\frac{N_{2}}{\widetilde{\tau}_{F}}-\frac{N_{2}^{2}}{N_{T} \widetilde{\tau}_{Q}}
$$

It must be used to simulate heat generated with the quantum defect. Unavoidable impurities in the laser host material result in background absorption, which can be described by the background absorption coefficient $\alpha_{B}(\lambda)$. Even small background absorption can significantly deteriorate the laser cooling process. The heat power density generated in the laser by quenching can be described as

$$
P_{\text {heat }}^{Q}=\hbar \omega_{P} N_{T} \widetilde{N}_{2}\left(\frac{1}{\tau_{F}}-\frac{1}{\tau_{R}}+\frac{\widetilde{N}_{2}}{\widetilde{\tau}_{Q}}\right)
$$

where

$$
\widetilde{N}_{2} \approx \frac{N_{2}}{N_{T}}\left(1+\frac{\left(1-\tau_{R} / \widetilde{\tau}_{F}\right)-N_{2} \tau_{R} /\left(N_{T} \widetilde{\tau}_{Q}\right)}{\widetilde{I}_{P}+\widetilde{I}_{L}+\tau_{R} / \widetilde{\tau}_{F}}\right),
$$

and

$$
\widetilde{I}_{P, L}=I_{P, L} \frac{\tau_{R}\left(\sigma_{a}^{P, L}+\sigma_{e}^{P, L}\right)}{\hbar \omega_{P, L}} .
$$

The heat power density caused by background absorption has the form

$$
P_{\text {heat }}^{a}=\frac{\hbar}{\tau_{R}}\left(\frac{\widetilde{i}_{P} \omega_{P} \alpha_{B}\left(\lambda_{P}\right)}{\sigma_{a}^{P}+\sigma_{e}^{P}}+\frac{\widetilde{i}_{L} \omega_{L} \alpha_{B}\left(\lambda_{L}\right)}{\sigma_{a}^{L}+\sigma_{e}^{L}}\right),
$$


where $\widetilde{i}_{P, L}=I_{P, L} / \widetilde{I}_{P, L}$. These values must be added to the heat power density generated with the quantum defect if one wants to simulate the heat power density generated by different effects in the realistic laser system.

Summarizing the theory of radiation-balanced lasing, it is important to emphasize that laser materials suitable to radiation-balanced lasing must have two widely separated sets of discreet energy levels (manifolds) such that nonradiative transitions between them are unlikely. They also must have negligible background absorption. A properly arranged reliable pump system, the shape of a laser gain medium, and material selection criteria can result in little or no heat generation within solid-state lasers.

\section{Development of Radiation-Balanced Lasers}

The thermodynamics of radiation-balanced lasers has been discussed both qualitatively and quantitatively from the point of view of the first and second laws by Mungan [28]. The Carnot efficiency has been derived for optical amplification from consideration of the radiative transport of energy and entropy. It has been shown that the highest Carnot efficiencies can be reached only when the system is pumped into saturation. Indeed, the energy of a collection of photons of fixed mean frequency and angular divergence scales linearly with the number of photons. The entropy of the set depends on the number and on the energy distribution of the photons. As the number of photons in the pump beam increases, the entropy that the beam carries per photon decreases since it becomes more sharply peaked. As the fluorescence intensity increases, the entropy decreases, but it does not decrease as rapidly as does the pump beam, because the spontaneously emitted light is less intense, broader in bandwidth, and distributed over a larger range of solid angles than is the pump radiation.

As one can see from the theory developed in the previous part of the paper, a detailed balance of the stimulated and spontaneous emission at each point of the laser medium can provide a solid-state laser that generates no internal heat. Unfortunately, there are two serious problems associated with the practical development of radiation-balanced amplifiers and lasers: the precise control of the pump power and almost linear growth of the amplified signal (Figure 5). The linear growth of the power of the amplified signal requires an enormous increase in the length of the active medium for very high output power. The athermal bulk or fibre laser requires precision control of the pump power at each point along the length laser medium. This is not a simple problem, especially in the case of a fibre amplifier or laser. The sensitivity and stability of a radiation-balanced laser to perturbations in the field parameters and temperature have been analyzed in [29]. As one can see in [29], fluctuations in the gain set limits on the variability of the pump wavelength. A pump stability of $\pm 1 \mathrm{~nm}$ was suggested for an $\mathrm{Yb}: \mathrm{KGW}$ laser. An active wavelength stabilization scheme based on techniques for spatial mode matching was presented in [29]. A dynamic control to the laser system based on the pump wavelength shift was proposed. It permits to minimize the sensitivity of the athermal laser to ambient temperature fluctuations. The idea to use tapered reflectance mirrors to flatten the transverse laser profile and to improve optical efficiency was considered in [29]. 


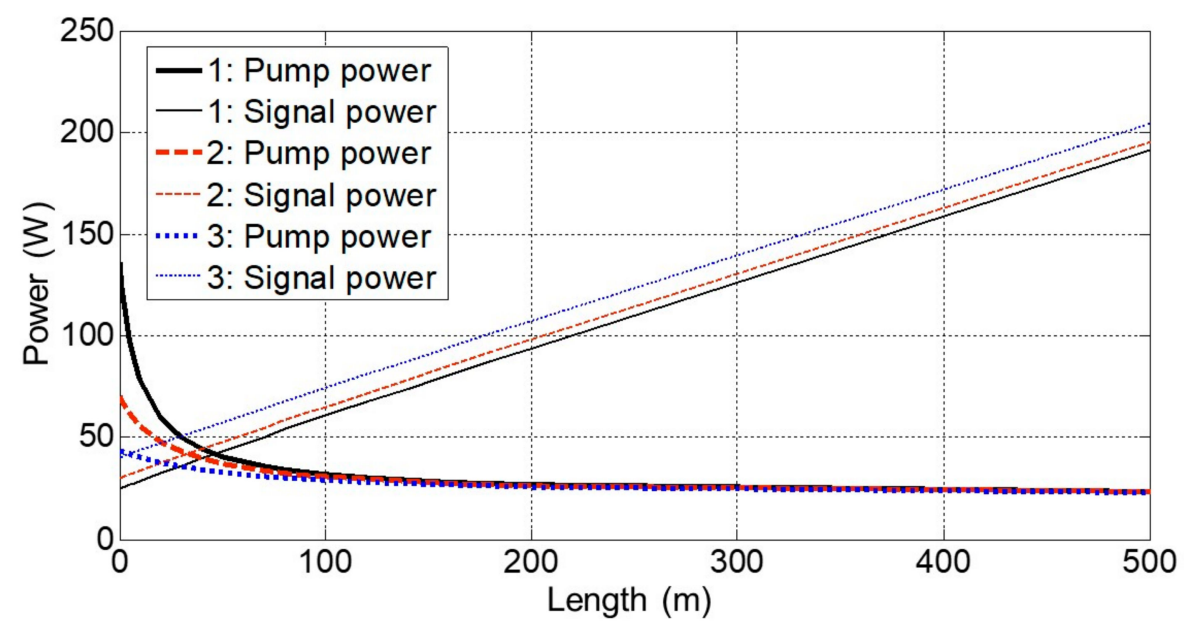

Figure 5. Dependence of the signal and pump powers from the length of the athermal fibre amplifier for three different input signal powers: (1) $25 \mathrm{~W}$, (2) $30 \mathrm{~W}$, and (3) $40 \mathrm{~W}$ [26].

\subsection{Athermal Bulk Lasers}

Since the first experimental observation in 1995, laser cooling of solids has been realized with $\mathrm{Yb}^{3+}, \mathrm{Er}^{3+}, \mathrm{Tm}^{3+}$, and $\mathrm{Ho}^{3+}$ ions doped in a wide variety of low phonon glasses and crystals. $\mathrm{Yb}^{3+}$ ions are the most promising RE ions for laser cooling applications. They have only one excited manifold, so that $\mathrm{Yb}^{3+}$ ions are free from excited state absorption, which can be a source of multi-phonon decay resulting in undesirable heat generation in the system.

Experiments devoted to radiation-balanced lasers began at the Naval Research Laboratory (NRL) in 1999 [30]. New materials for laser cooling were investigated. In 2002, the first athermal bulk laser was experimentally demonstrated with an $\mathrm{Yb}^{3+}: \mathrm{KGd}\left(\mathrm{WO}_{4}\right)_{2}$ crystal [31]. The laser design based on direct diode pumping of $\mathrm{Yb}^{3+}: \mathrm{KGd}\left(\mathrm{WO}_{4}\right)_{2}$ crystals was developed further in [32].

Successful laser systems are based on high-quality materials. YAG and fused silica are the best choice. They have the highest optical quality and lowest losses. In 2010, the $\mathrm{Yb}^{3+}$ :YAG laser rods with $\mathrm{Yb}^{3+}$ concentrations of $1 \%, 2 \%, 3 \%$, and $5 \%$ were investigated.

The scheme of the radiation-balanced laser based on the $\mathrm{Yb}^{3+}$ :YAG laser rod is presented in Figure 6. High power pumping with fibre lasers at $1030 \mathrm{~nm}$ produced a near diffraction limited laser at $1050 \mathrm{~nm}$. The mean fluorescent wavelength for $\mathrm{Yb}^{3+}: Y A G$ is $1010 \mathrm{~nm}$. Lower thermal loading and better beam quality were reached with the $2 \%$ $\mathrm{Yb}^{3+}$ :YAG rod. The average power of up to $200 \mathrm{~W}$ of output with a beam quality of $\mathrm{M}^{2}=1.2$ was demonstrated without significant thermal loading or thermo-optic distortions [33]. A typical thermal image of the $\mathrm{Yb}^{3+}: Y A G$ rod inside the athermal laser is presented in Figure 7. Thermal loads were reduced to below $0.01 \%$ relative to the laser output power. As one can see in Figure 7, fluorescence for the laser rod heats the mirror on the left. The thermally insulated laser rod is almost not heated. Its temperature is very close to the ambient temperature. 


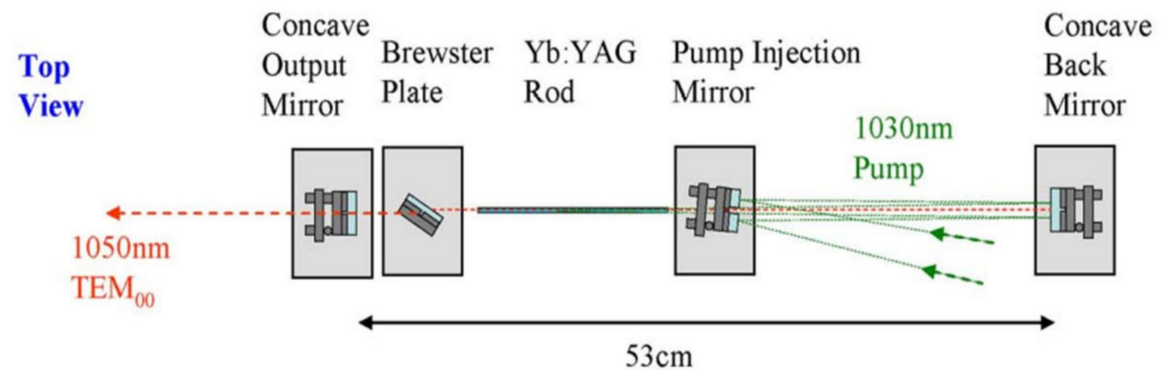

(a)

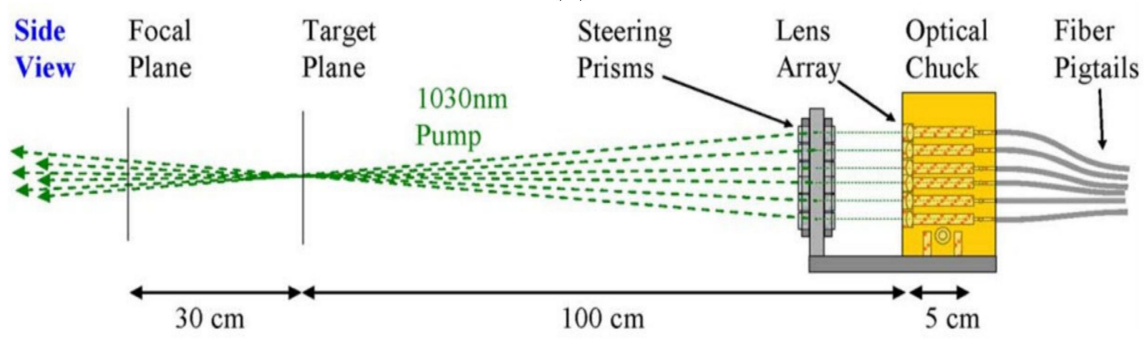

(b)

Figure 6. Athermal laser: (a) Stable $\mathrm{Yb}^{3+}$ :YAG resonator. (b) Ytterbium fibre laser pump array propagation shown without the target laser rod [34].

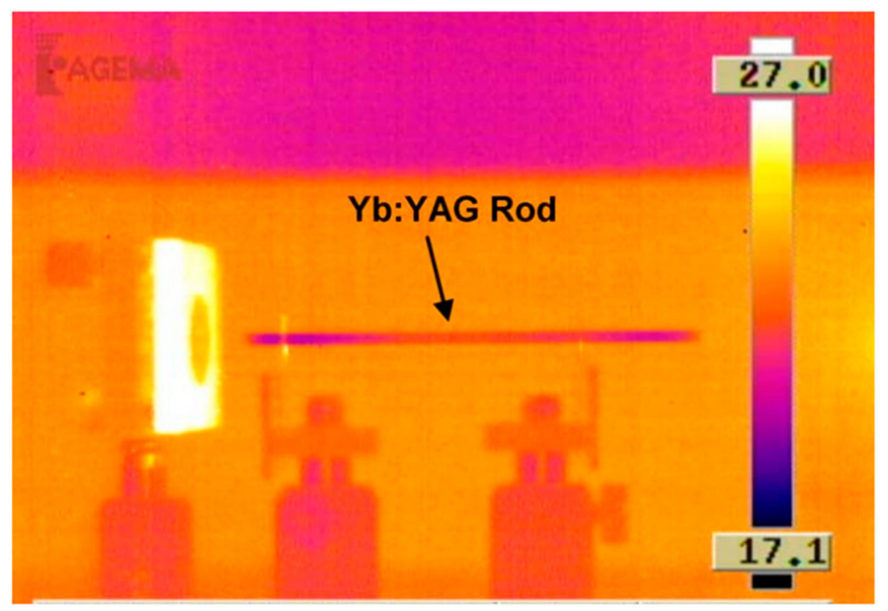

Figure 7. $\mathrm{Yb}^{3+}:$ YAG rod inside the laser. This image was captured after $10 \mathrm{~s}$ of lasing at $70 \mathrm{~W}$ with an ambient temperature of $22{ }^{\circ} \mathrm{C}$. The color scale corresponds to $\mp 5{ }^{\circ} \mathrm{C}$ as shown on right. Fluorescence from the laser rod heats the mount for the pump injection mirror on left [33].

To improve laser performance, the laser rod was replaced with a slab composed of $\mathrm{Yb}^{3+}: Y A G$ and sapphire (Figure 8) [34]. As one can see in Figure 8, a single $2 \mathrm{~mm} \times$

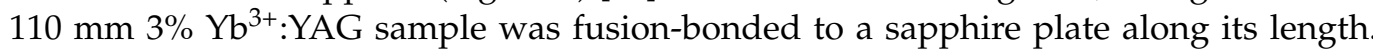
The sapphire plate provides a refractive index mismatch of 0.07 , significantly reducing fluorescence trapping and providing guiding of the pump at the wavelength $1030 \mathrm{~nm}$. The sapphire bond permitted to reduce the fluorescence lifetime from $2 \mathrm{~ms}$ to $1.25 \mathrm{~ms}$. Using this laser design, $1 \mathrm{~W}$ of heat for every kilowatt of output laser power was realized.

\subsection{Athermal Disk Lasers}

In 2000, an $\mathrm{Yb}^{3+}: \mathrm{KGW}$ laser disk was edge-pumped with radially focused laser diode bars (Figure 9a) [34]. It generated up to $490 \mathrm{~W}$. This was the first system with heat loading below the laser quantum defect. 


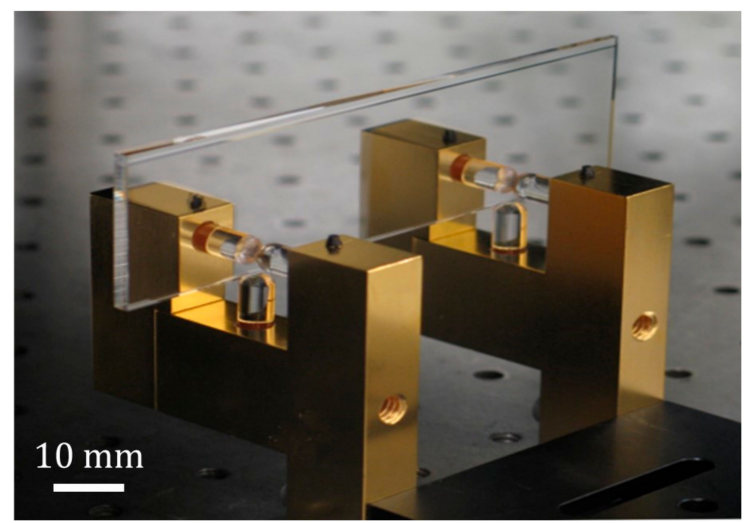

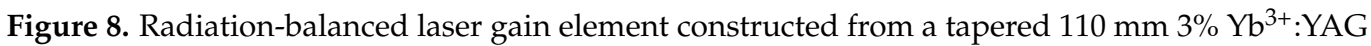
slab fusion-bonded to a $2 \mathrm{~mm} \times 20 \mathrm{~mm}$ sapphire plate. The sapphire plate provides a refractive index mismatch of 0.07 . This allows for guiding of the $1030 \mathrm{~nm}$ pump lasers while significantly reducing fluorescent trapping. The gold-plated mount and fused silica pins were designed to avoid radiative heating of the fixture [34].

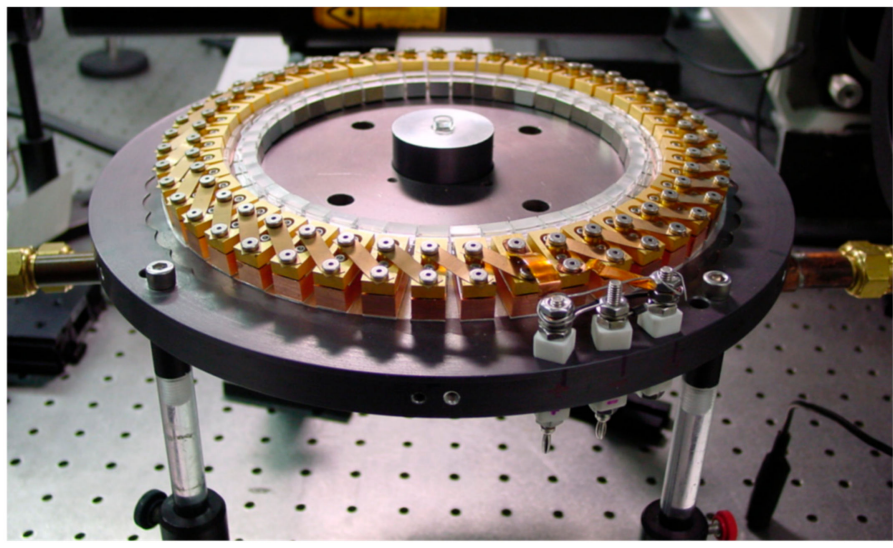

(a)

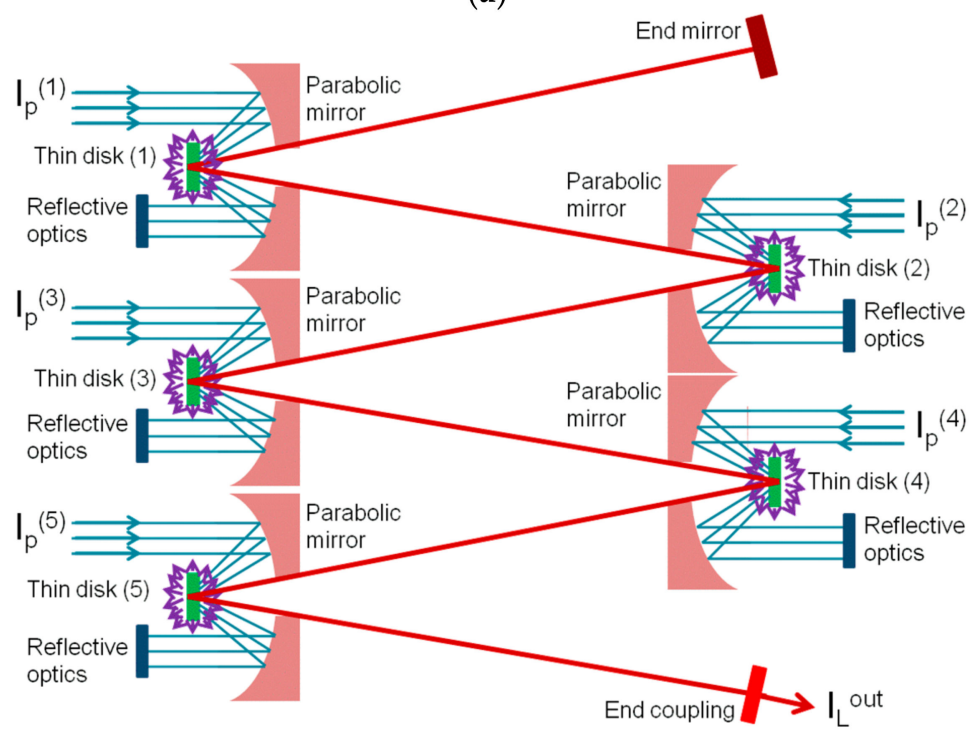

(b)

Figure 9. $\mathrm{Yb}^{3+}: \mathrm{KGW}$ disk lasers. (a) Edge-pumped $\mathrm{Yb}^{3+}: \mathrm{KGW}$ disk laser with a laser quantum defect of $4.8 \%$. (b) Thin-disk athermal laser system. $\mathrm{I}_{\mathrm{p}}{ }^{(1)}, \mathrm{I}_{\mathrm{p}}{ }^{(2)}, \mathrm{I}_{\mathrm{p}}{ }^{(3)}, \mathrm{I}_{\mathrm{p}}{ }^{(4)}$, and $\mathrm{I}_{\mathrm{p}}{ }^{(5)}$ are the pump intensities, $\mathrm{I}_{\mathrm{L}}$ out is the intensity of the output laser signal. The violet arrows around the disks illustrate anti-Stokes fluorescence [35]. 
In 2014, a thin-disk athermal laser system was proposed and theoretically analysed [35]. This scheme, consisting of a sequence of thin disks, can provide high flexibility, which is key for athermal laser operation (Figure $9 b$ ). $\mathrm{Yb}^{3+}: \mathrm{KGW}$ laser disks were considered in [35]. The sequence of thin disks provides precise control of the pump intensity on each disk. It allows the control of the pump intensity almost locally; that is, almost at each point along all laser gain medium. In addition, the generic design of the laser module allows easy scaling to higher powers. The concentration of the ions, as well as the thickness, can be changed from one disk to another.

In 2019, the radiation-balanced $\mathrm{Yb}^{3+}: \mathrm{YAG}$ disk laser was experimentally demonstrated in an intracavity pumping geometry (Figure 10) [36]. An optically pumped vertical-externalcavity surface-emitting laser (VECSEL) was used to enhance the pump absorption. The broad tunability and good beam quality of VECSELs provided additional parametrical freedom (Figure 10).

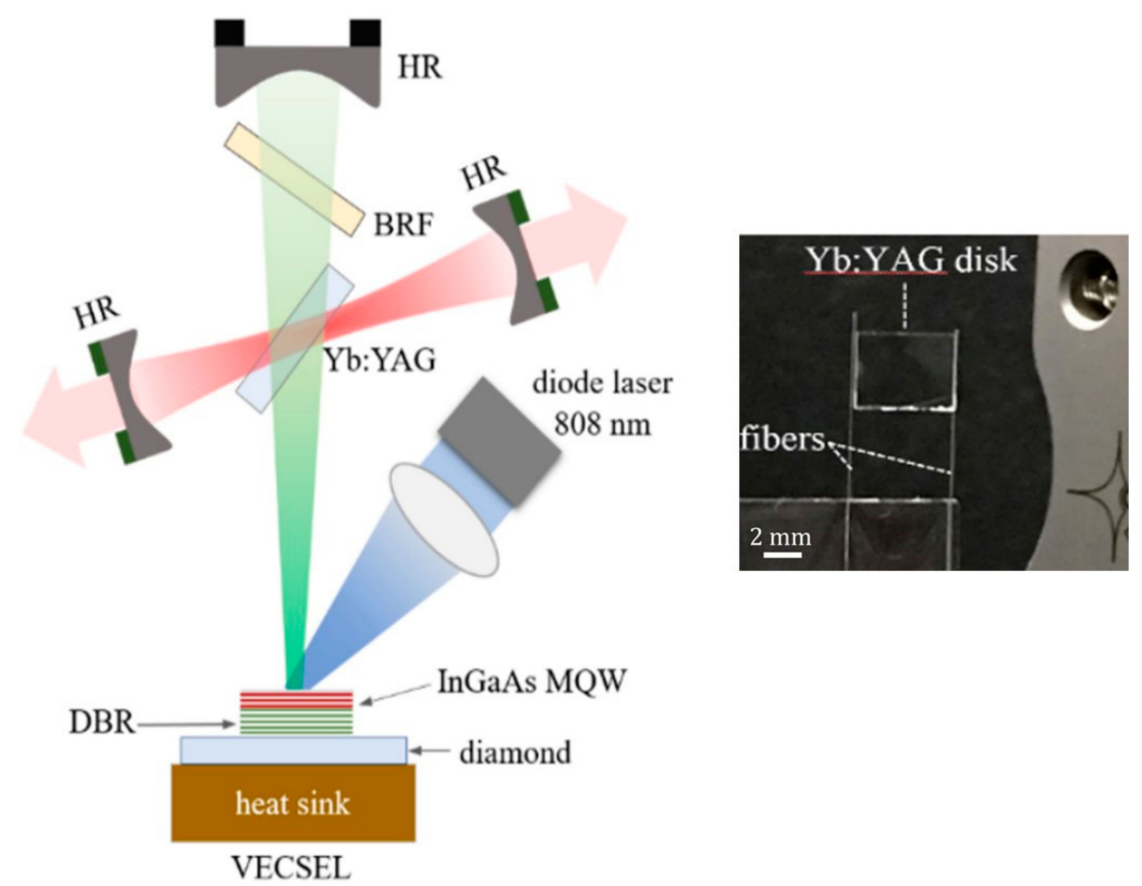

Figure 10. Schematic of the intracavity-pumped radiation-balanced disk laser setup. On the right, the mounting of the $5 \%$-doped $\mathrm{Yb}^{3+}: \mathrm{YAG}$ disk of $0.5 \mathrm{~mm}$ thick and $4 \mathrm{~mm} \times 5 \mathrm{~mm}$ cross section is shown where it is glued onto two bare fibres, which are in turn supported by a glass slide to reduce the heat load [36].

The thermal images of the mounted laser disk at room temperature after $30 \mathrm{~min}$ at radiation-balanced condition are presented in Figure 11. Darker shades represent lower temperatures. Recorded thermal images of the unpumped laser disk at room temperatures, and under pump, are shown in Figures 11a and 11b, respectively. Figure 11c illustrates the temperature dynamics of the radiation-balanced process. The temperature profile of the laser disk was monitored with a thermal camera (Nanocore 640, L3 Communications Corporation, Garland, TX, USA) with $0.05 \mathrm{~K}$ resolution as the VECSEL power was varied. As one can see in Figure 11c, net cooling takes place from the very beginning, that is, as soon as the pump diode is turned on. Indeed, the VECSEL and the fluorescence emission process start immediately. The lasing process with the $\mathrm{Yb}^{3+}$ :YAG disk takes some time to initiate. It warms up the laser disk. About $10 \%$ power fluctuation was observed in the first $5 \mathrm{~min}$ of the experiment. The radiation balancing condition was realized with $57 \mathrm{~W}$ of incident pump power at $808 \mathrm{~nm}$, or $32 \mathrm{~W}$ of absorbed power. About $1 \mathrm{~W}$ output power radiation balance was obtained. A temperature difference of $\sim 2 \mathrm{~K}$ was observed between the centre and the edge of the disk of $4 \mathrm{~mm} \times 5 \mathrm{~mm}$ cross section as a result of 
Gaussian radial intensity distribution of the modes. Beam shaping can be used to mitigate this temperature difference.

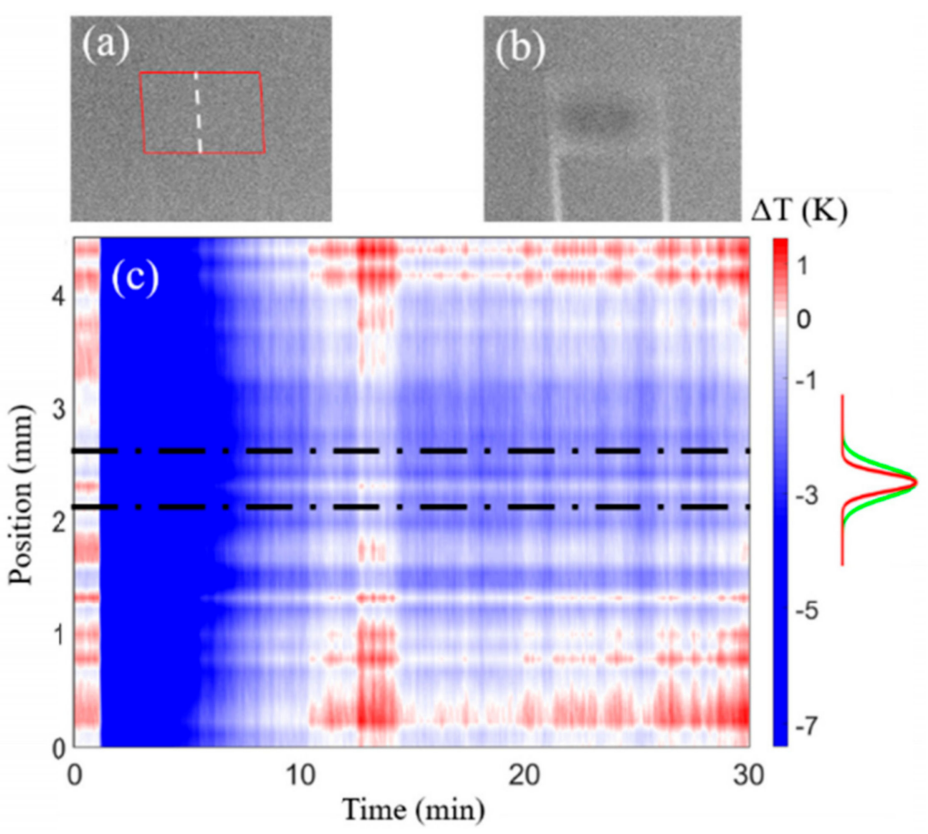

Figure 11. The thermal images of the laser disk; (a) is the unpumped laser disk at room temperature. The red lines represent the outline of the disk; (b) is the laser pumped disk. (c) The line-integrated time-evolution of the temperature change along the white dashed line in (a). The VECSEL cavity is unblocked at $1 \mathrm{~min}$ [36].

Recently, $\mathrm{Yb}^{3+}: \mathrm{YLF}$ and $\mathrm{Yb}^{3+}: \mathrm{LLF}$ laser disks have been investigated in external multipass pumping schemes (Figure 12) [37,38]. Compared to intracavity pumping, external multipass pumping offers higher control on pump spot size and mode-matching conditions. LLF is an isomorph of YLF. They have very similar thermo-optics properties. The $\mathrm{Yb}^{3+}: \mathrm{YLF}$ and $\mathrm{Yb}^{3+}$ :LLF laser disks with the $\mathrm{Yb}^{3+}$ concentration of $10 \%, 1 \mathrm{~mm}$ thick, and $5 \mathrm{~mm}$ diameter were pumped at the wavelength $1020 \mathrm{~nm}$ corresponding to the lowest energy transition between ground and excited manifolds of $\mathrm{Yb}^{3+}$ ions. This wavelength is convenient for the system cooling. Laser emission was observed around $1050 \mathrm{~nm}$. The linear laser cavity is constituted by mirrors M1 and M3, both with $10 \mathrm{~cm}$ radius of curvature (Figure 12a). A hole in the center of the M2 mirror allows propagation of the laser mode. The input laser beam was focused near the focal point of M1 and M2, and thus collimated after reflection of M2 and focused again after reflection on M1. A tilted window at Brewster's angle was used to shift the beam towards the center of M2. After several roundtrips between M1 and M2, the pump is eventually scattered out by the sidewalls of the window. The sample was placed at the middle point of M1 and M2. Radiation-balanced operation at about $1 \mathrm{~W}$ output power was achieved with the $\mathrm{Yb}^{3+}$ :YLF disk. Radiation-balanced laser operation was observed at about $200 \mathrm{~mW}$ output power with the $\mathrm{Yb}^{3+}$ :LLF laser disk (Figure 12b). It was shown that large transversal thermal gradients are expected in beam area for high-power $(\mathrm{kW})$ radiation-balanced laser operations that require mitigation techniques. 
(a)

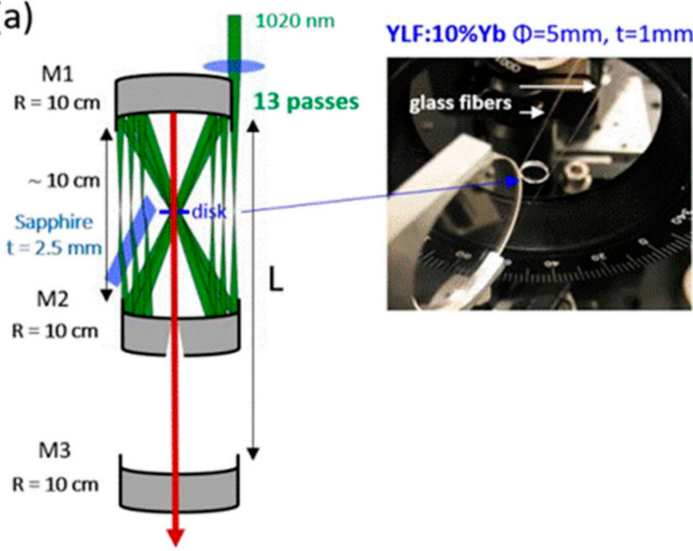

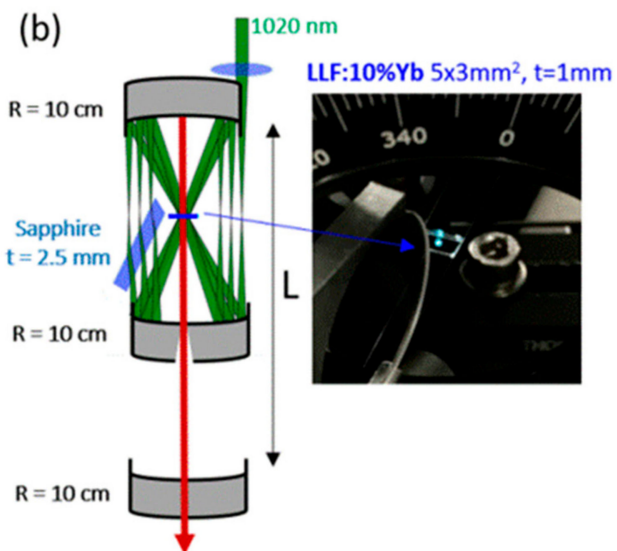

Figure 12. Schematic of laser cavity and multipass pumping for (a) the $\mathrm{Yb}^{3+}: \mathrm{YLF}$ laser disk and for $(\mathbf{b})$ the $\mathrm{Yb}^{3+}: \mathrm{LLF}$ laser disk [38].

\subsection{Athermal Fibre Lasers}

As previously noted, in radiation-balanced lasers and amplifiers, the radiated energy increases only linearly with the length of the active medium. This requires a long active medium, which is undesirable. To overcome the problem, lasers and amplifiers with a cooler made from RE ions and integrated in the body of the device have been proposed and theoretically investigated by Nemova and Kashyap [39-41].

As one can see in Figure $13 \mathrm{a}$, the properly distributed $\mathrm{Yb}^{3+}$ ions pumped at the wavelength $1015 \mathrm{~nm}$ with a cooling pump power $P_{p}{ }^{\text {cool }}$ work as an integrated cooler, compensating for the heat generated inside the active medium due to the quantum defect in the Raman lasers or amplifiers. In this scheme, the lasing action is separated from the cooling process. Such separation of actions provides flexibility in the laser design. It permits one to maintain the exponential growth of the amplified signal along the length of the laser medium.

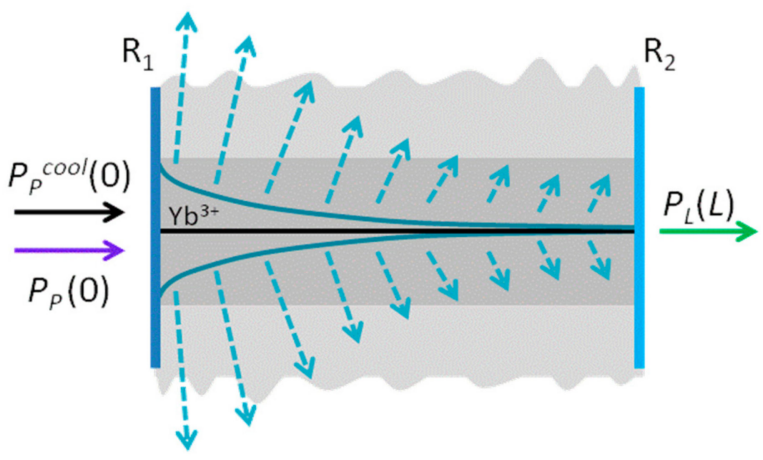

(a)

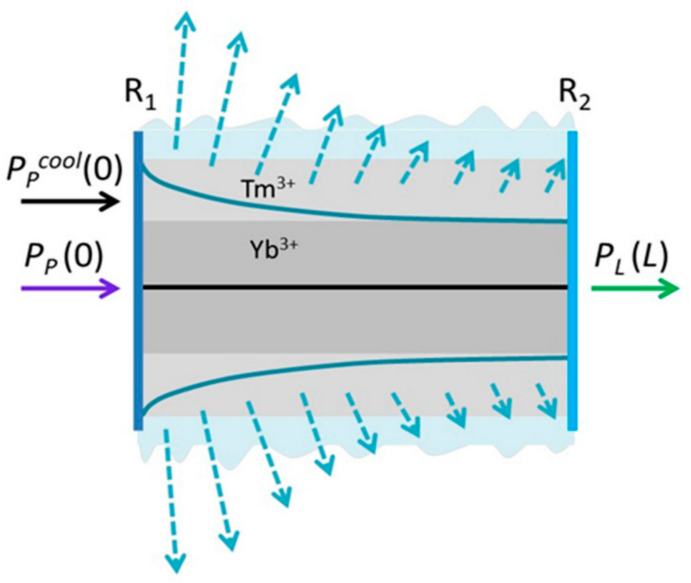

(b)

Figure 13. Fibre lasers with an integrated cooler. (a) Athermal Raman fibre laser. Two curves in the core illustrate the distribution of $\mathrm{Yb}^{3+}$ ions providing cooling. (b) Athermal $\mathrm{Yb}^{3+}$-doped fibre laser with the Tm ${ }^{3+}$-doped cladding. Two curves in the cladding illustrate the distribution of $\mathrm{Tm}^{3+}$ ions providing cooling. The dashed arrows illustrate anti-Stokes fluorescence [41].

Figure $13 \mathrm{~b}$ illustrates an athermal $\mathrm{Yb}^{3+}$-doped fibre laser, in which the heating is caused by Stokes-shifted stimulated emission generated during the lasing process. In this scheme, the lasing process takes place in the $\mathrm{Yb}^{3+}$-doped fibre core. The cooling process takes place in the $\mathrm{Tm}^{3+}$-doped fibre cladding pumped at a wavelength of $1900 \mathrm{~nm}$, that 
is, in the long-wavelength tail of the $\mathrm{Tm}^{3+}$ absorption spectrum. Here, the $\mathrm{Tm}^{3+}$ ions play the role of an integrated cooler. The distribution of the RE ions in the integrated cooler is properly arranged in both schemes to provide complete compensation for the heat in the case of fixed laser pump power, $P_{p}$. The dashed arrows in Figure 13 illustrate anti-Stokes fluorescence. A deviation in the laser input pump power causes a small deviation in complete compensation of the temperature along the length of the laser medium. However, this deviation in the temperature is small in comparison with the peak temperature change without cooling. It can be partially compensated for by a change in the cooling pump power, $P_{p}{ }^{\text {cool. }}$.

The temperature distribution inside a double-cladding optical fibre laser or amplifier was theoretically investigated by Mafi [42]. It has been shown that heat generation in the cladding due to the parasitic absorption of the high-power pump must be taken into account in modern high-power fibre lasers and amplifiers, where the quantum defect is lowered, or when the amplifier operates in a nearly radiation-balanced regime, or for radiation-balanced lasers. The developed approach permits one to interpolate between the case where the quantum defect heating is dominant and when the parasitic absorption heating is comparable in size or is the dominant source of heating.

\subsubsection{Athermal ZBLAN Fibre Lasers}

An ytterbium-doped ZrF4-BaF2-LaF3-AlF3-NaF (ZBLAN) optical fibre was experimentally investigated to extract its laser cooling related parameters [43]. It has been shown that the $\mathrm{Yb}^{3+}:$ ZBLAN fibre is a viable laser-cooling medium for radiation-balanced lasers and amplifiers. A comparison between a conventional $\mathrm{Yb}^{3+}:$ ZBLAN fibre laser pumped at $975 \mathrm{~nm}$ wavelength and an athermal $\mathrm{Yb}^{3+}:$ ZBLAN fibre laser pumped at $1030 \mathrm{~nm}$ was made [43]. They are presented in Figure 14. The output laser signal in both lasers was assumed to be $3 \mathrm{~W}$ in power at the wavelength $1070 \mathrm{~nm}$. Both systems were optimized for maximum efficiency. As one can see in Figure 14, the temperature rise in both designs is comparable, while the athermal laser required a 20-fold larger pump power, making the athermal laser design totally unpractical. The authors of [43] attributed the problem to the relatively large value of the background absorption of the pump. They considered the problem of the background absorption in [44]. Following their simulations, a 10-fold reduction in the background absorption can reduce the heating in the athermal laser design significantly, rendering the athermal laser viable. These results are presented in Figure 14.

In 2021, the first observation of anti-Stokes cooling of $\mathrm{Yb}^{3+}:$ ZBLAN fibres, in which both the core and the cladding were doped with $\mathrm{Yb}^{3+}$ to increase the number of $\mathrm{Yb}^{3+}$ ions contributing to cooling, was announced [45]. It is worth mentioning that laser cooling of the core-doped $\mathrm{Yb}^{3+}:$ ZBLAN fibres was demonstrated previously in a vacuum [46] and at atmospheric pressure [47,48]. The main goal of [45] was to provide an experimental validation of the concept of cooling a fibre more aggressively by doping its cladding with a cooling ion and pumping the cladding, which was proposed by Nemova and Kashyap in [39]. The performances of three fibre designs were compared. One of these fibres was a conventional large-core multimode fibre. Two others were cladding-doped fibres. One of these two cladding-doped fibres had a double-D shape, and the other had an octagonal cladding. Both shapes were designed to induce greater mode mixing (Figure 15). The pump light from a $1025.5 \mathrm{~nm}$ fibre-pigtailed semiconductor laser was launched into the $\mathrm{Yb}^{3+}$ :ZBLAN fibre under test. The temperature change of a fibre was measured with a custom slow-light FBG sensor with a sub-mK resolution [49]. 
(a) left

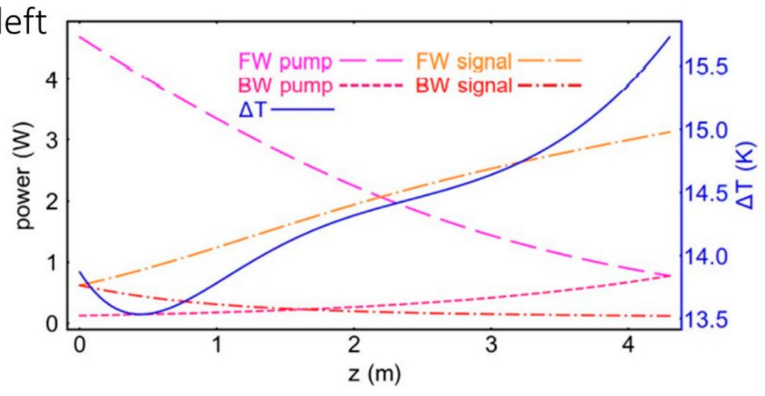

(b) left

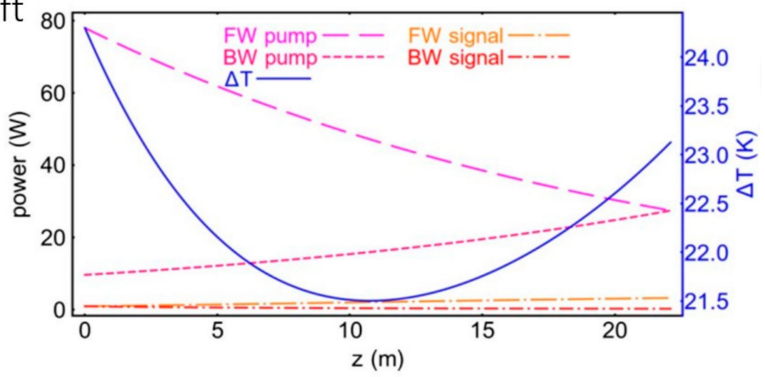

(a) right

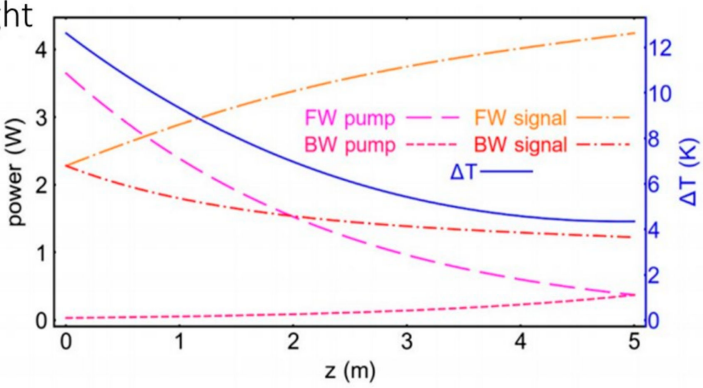

(b) right

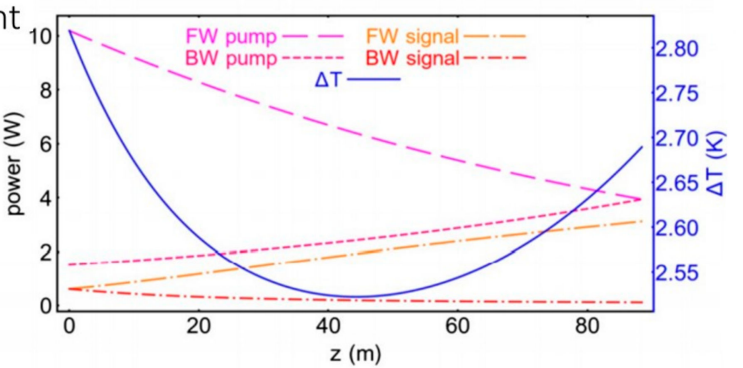

Figure 14. (a) Propagation of the forward pump (FW pump), backward pump (BW pump), forward signal (FW signal), backward signal (BW signal), and temperature rise along the fibre for a conventional fibre laser pumped at $975 \mathrm{~nm}$. (b) Similar graph for the athermal laser operation pumped at $1030 \mathrm{~nm}$. In $(\mathbf{a}, \mathbf{b})$ on the left, the background absorption is $4.278 \times 10^{-2} \mathrm{~m}^{-1}$. In $(\mathbf{a}, \mathbf{b})$ on the right, a 10-fold reduction is shown in the background absorption $[43,44]$.

(a)

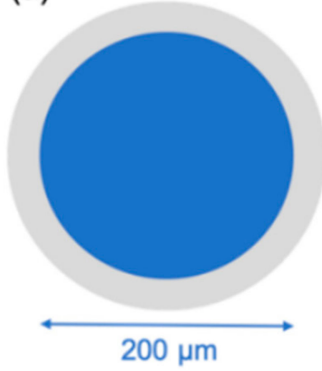

(b)

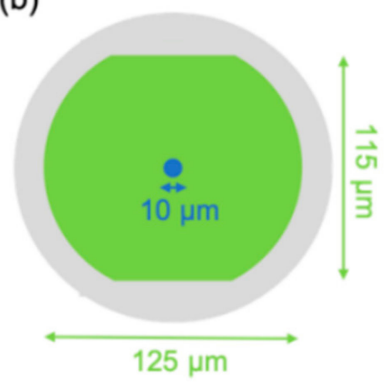

(c)

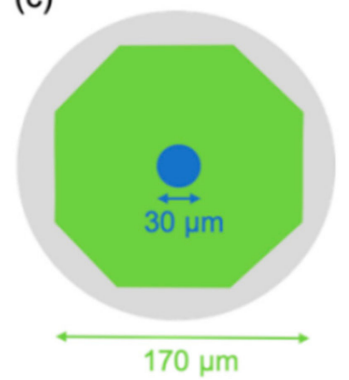

Figure 15. Cross section of the multimode Yb-doped ZBLAN fibres used in [45]: (a) conventional multimode fibre, (b) double-D cladding-doped fibre, and (c) cladding-doped fibre with an octagonal inner cladding [45].

The conventional multimode fibre (Figure 15a) with relatively poor mode mixing due to its symmetrical core was used to illustrate the importance of mode mixing. The maximum measured temperature change observed in this fibre under $1025.5 \mathrm{~nm}$ pumping was $-0.65 \mathrm{~K}$ for $584 \mathrm{~mW}$ of pump power at the location of the sensor.

In order to improve mode mixing, a second fibre with a double-D cladding doped with $\mathrm{Yb}^{3+}$ was fabricated (Figure 15b). The largest temperature change recorded for this fibre was $-78 \mathrm{mK}$ for a pump power of $240 \mathrm{~mW}$. The efficiency (temperature change divided by pump power) was higher ( $\sim 3$ times) than in the conventional multimode fibre.

The third $\mathrm{Yb}^{3+}:$ ZBLAN fibre, which had an octagonal cladding, was designed specifically to improve the mode filling of the cladding (Figure 15c). This fibre design permits the mode to fill a noticeably larger portion of the cladding. The temperature change of $-1.3 \mathrm{~K}$ was reached with the maximum available pump power of $3 \mathrm{~W}$. To improve this value, the fibre was pumped with the pump power of $15 \mathrm{~W}$ at the wavelength $1040 \mathrm{~nm}$. The temperature change of $-2 \mathrm{~K}$ was reached.

The fibre with a double-D cladding (Figure 15b) did not perform as well as the fibre with an octagonal inner cladding (Figure 15c), largely due to limited mode mixing, which 
resulted in only a small fraction of the ions in the cladding being excited and contributing to the refrigeration process. An octagonal inner cladding of the fibre (Figure 15c) permitted to increase mode mixing and excite the cladding more uniformly. As a result, greater cooling was observed with this fibre.

\subsubsection{Athermal Silica Fibre Lasers}

Most commercial fibre lasers are made of silica, due to silica's low loss and durability. Laser powers as high as $10 \mathrm{~kW}$ have been achieved with a silica fibre laser [50]. However, crystals and fluoride-based samples are widely used hosts for laser cooling with anti-Stokes fluorescence. Relative to silica, fluorides have fairly high loss and brittleness, that is, they can undergo sudden structural failure instead of plastic deformation. As a result, the highest power achieved with a ZBLAN laser is only $50 \mathrm{~W}$ [51].

In 2019, laser cooling with anti-Stokes fluorescence was demonstrated in two separate silica samples: a silica fibre [52] and a bulk silica preform [53]. These results were breakthroughs for development of silica-based athermal lasers. In [54], several influential factors on laser cooling with anti-Stokes fluorescence in silica fibres were investigated. The cooling performances of six $\mathrm{Yb}^{3+}$-doped silica fibres with various dopant and codopant concentrations, $\mathrm{OH}^{-}$loss, and core dimensions were compared. It was shown that silica can accommodate unexpectedly high $\mathrm{Yb}^{3+}$ concentrations without suffering from quenching, in spite of its high phonon energy. Cooling a fibre with high $2.52 \mathrm{wt} . \% \mathrm{Yb}^{3+}$ concentration was enabled by reducing the absorptive loss below $5 \mathrm{~dB} / \mathrm{km}$ and codoping the fibre with $2.0 \mathrm{wt} . \% \mathrm{Al}$, leading to a record-breaking critical quenching concentration, 30\% higher than the previous reported. A temperature change of $-70 \mathrm{mK}$ was observed at atmospheric pressure for $170 \mathrm{~mW} / \mathrm{m}$ of pump power absorbed at $1040 \mathrm{~nm}$.

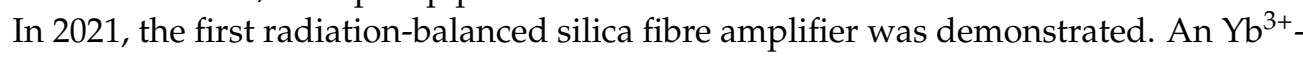
doped silica fibre served as an active medium [55]. The core diameter of the silica fibre was $21 \mu \mathrm{m}$. Its numerical aperture was 0.13 . The $\mathrm{Yb}^{3+}$ concentration was $2.52 \mathrm{wt} . \%$. The fibre was codoped with $2.00 \mathrm{wt} . \% \mathrm{Al}$ to reduce concentration quenching. The wavelength of the pump was $1040 \mathrm{~nm}$ and the signal wavelength was $1064 \mathrm{~nm}$. The mean fluorescence wavelength of $\mathrm{Yb}^{3+}$ was $1003.9 \mathrm{~nm}$ and the radiative and quenching lifetimes were $765 \mu \mathrm{s}$ and $38 \mathrm{~ms}$, respectively. The $\mathrm{Yb}^{3+}$ critical quenching concentration was $21.0 \mathrm{wt} . \%$. The absorptive loss of the fibre was $18 \mathrm{~dB} / \mathrm{km}$. The temporal trace of the temperature change in this fibre amplifier is illustrated in Figure 16. At time $t=0 \mathrm{~s}$, the pump was turned on. The temperature of the fibre decreased $\sim 130 \mathrm{mK}$ below room temperature. After $15 \mathrm{~s}$, the seed signal was turned on. The temperature of the fibre increased, reaching a new steady-state value of $\sim 110 \mathrm{mK}$ below room temperature. The temperature change of the fibre amplifier was defined as the difference between the average temperature in the first $5 \mathrm{~s}$, when both the pump and signal were off, and the average temperature in the last $5 \mathrm{~s}$ after both had been turned on. Each measurement was repeated three times and averaged. Measured temperature change versus position along the fibre amplifier is presented in Figure 17. The solid curves in Figure 17 are simulation results obtained using the model of a radiation-balanced fibre laser described in [56]. In [55], it was shown that light can be coherently amplified with a gain approaching $20 \mathrm{~dB}$ and generating no net internal heating. 


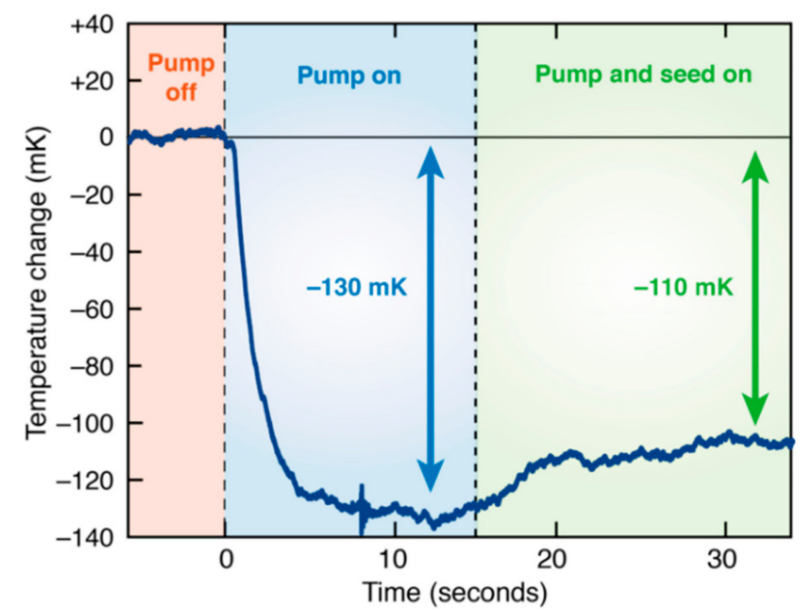

Figure 16. Measured temporal trace of the temperature change in the $\mathrm{Yb}^{3+}$-doped silica fibre as the $1040 \mathrm{~nm}$ pump and $1064 \mathrm{~nm}$ seed are sequentially turned on, launching $1.64 \mathrm{~W}$ and $3 \mathrm{~mW}$ in the fibre core, respectively [55].

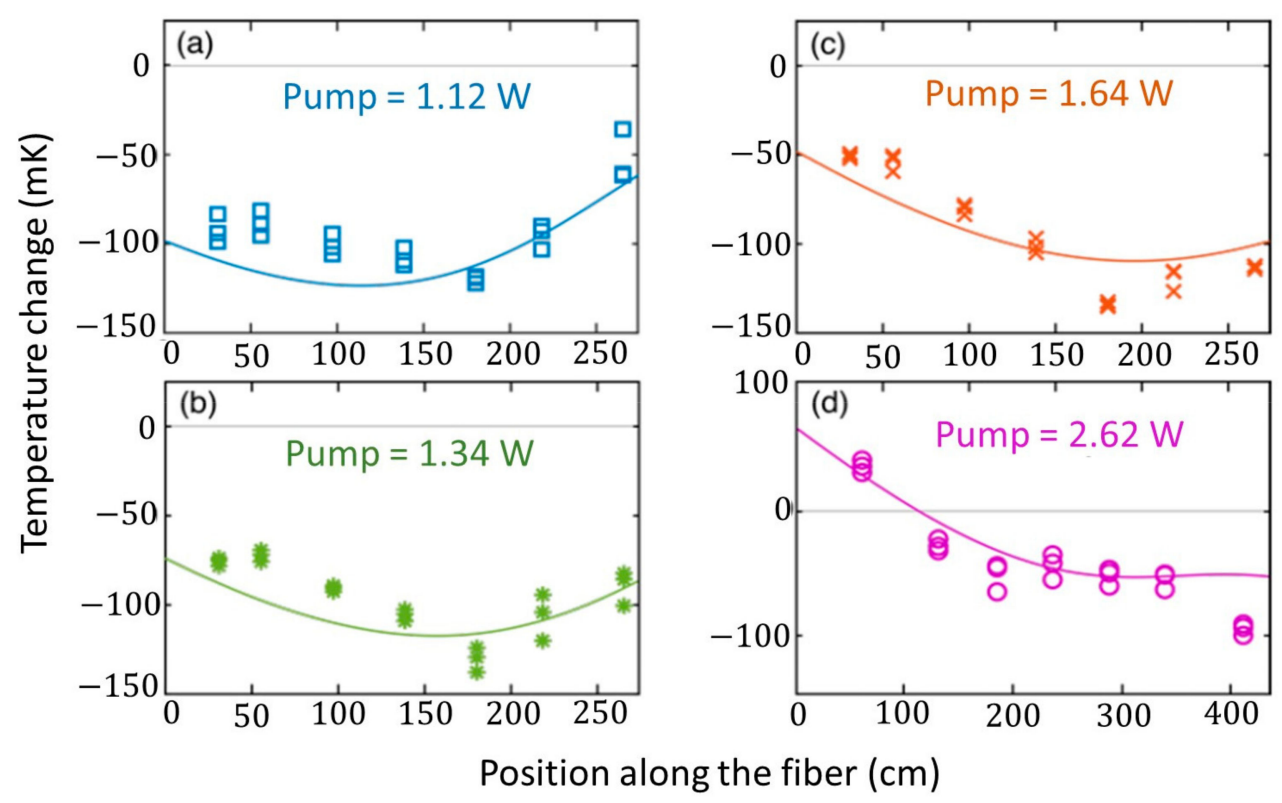

Figure 17. Measured temperature change versus position along the fibre amplifier, and simulated dependencies using the model based on [55]: for (a-c) a $2.74 \mathrm{~m}$, and (d) a $4.35 \mathrm{~m}$ amplifier fibre [55].

\subsubsection{Athermal Fibre Lasers with Optically Active Composite Cladding Materials}

The idea to use composite materials, such as glass-ceramic, for laser cooling with antiStokes fluorescence was proposed by Nemova and Kashyap in 2012 [57,58]. They proposed to use $\mathrm{Tm}^{3+}$-doped oxy-fluoride glass-ceramic to combine the low phonon energy of the $\mathrm{Tm}^{3+}$ doped fluoride nanocrystals with the promising mechanical and chemical properties of the oxide glass. In such a structure, $\mathrm{Tm}^{3+}$-doped nanocrystals serve as optically active sources of cooling.

Recently, the idea to use composite materials for optical cooling was experimentally developed and applied to fibre lasers by Xia et al. [59,60]. As a proof of principle, they demonstrated laser cooling of an YLF/polymer composite cladding material using far-field excitation [60]. As one can see in Figure 18a, a cladding-etched bare glass fibre with YLF crystals attached to it using a layer of commercially available fluoropolymer CYTOP with an ultra-low NIR absorption coefficient was illuminated perpendicularly in the far field 
with a $1020 \mathrm{~nm}$ continuous wave laser focused onto a crystal at a range of irradiances up to $1 \mathrm{MW} / \mathrm{cm}^{2}$. The pump laser at the wavelength $1020 \mathrm{~nm}$ excites electrons from energy level $\mathrm{E}_{4}$ to energy level $\mathrm{E}_{5}$. After thermalization accompanied by phonon absorption in the ${ }^{3} \mathrm{~F}_{5 / 2}$ manifold, electrons undergo transition to the ${ }^{3} \mathrm{~F}_{7 / 2}$ manifold, and the fluorescence spectrum arises (Figure 18b). The fluorescence spectra were recorded with a spectrometer (Ocean Optics, NIR512, Midland, ON, Canada). A 1000 nm short-pass filter (Thorlabs, FESH1000, Newton, MA, USA) was used to filter the laser line. Ten spectra, collected for $100 \mathrm{~ms}$ each, were averaged to obtain the final fluorescence spectrum. The temperature-calibrated fluorescence spectra were obtained using a cryostat (Janis, ST500, Westerville, OH, USA) in which the sample temperature was maintained at various points from $300 \mathrm{~K}$ to $350 \mathrm{~K}$ by a temperature controller (Lake Shore, 335, Westerville, OH, USA) with resolution $0.01 \mathrm{~K}$. Fluorescence spectra were normalized to the peak at $960 \mathrm{~nm}$. P1 and P2 are electronic transitions E6-E2 and E5-E2/E3, respectively. The integrated intensity ratio of P1 and $\mathrm{P} 2$ peaks is temperature dependent following a Boltzmann distribution (Figure 18c). The integration ranges for P1 and P2 are from $952 \mathrm{~nm}$ to $968 \mathrm{~nm}$ and from $986 \mathrm{~nm}$ to $1000 \mathrm{~nm}$, respectively. It was shown that the temperature of the $\mathrm{Yb}^{3+}$ :YLF crystal decreases by $6.6 \mathrm{~K}$ (Figure 18c). At each laser irradiance, a mean P1/P2 ratio was obtained by averaging six measurements, and the error bars represent one standard deviation, which are smaller than $1 \%$ of the mean values.

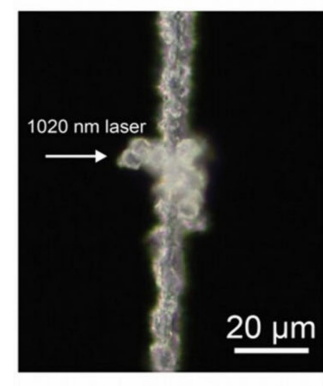

(a)

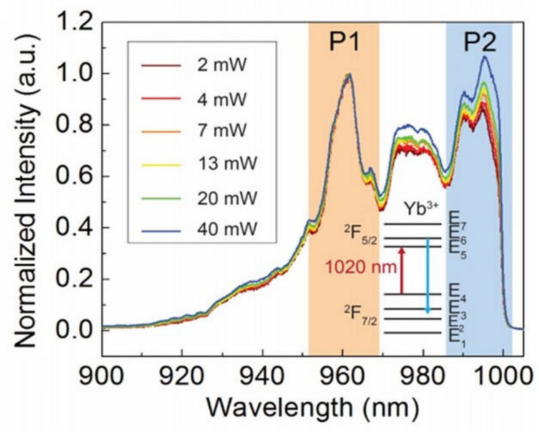

(b)

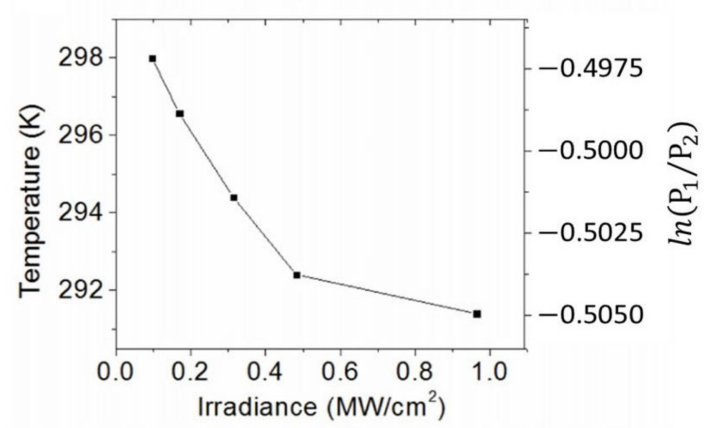

(c)

Figure 18. (a) Optical microscope image of $\mathrm{Yb}^{3+}: Y L F$ microcrystals on an etched fibre. (b) $\mathrm{Yb}^{3+}$ :YLF fluorescence spectra at various laser irradiances, normalized to the P1 peak. At higher irradiance the P2 peak increases in intensity. (c) The integrated intensity ratio of $\mathrm{P} 1$ and $\mathrm{P} 2$ peaks with the corresponding calibrated temperature at each laser irradiance [60].

In [60], Xia et al. proposed and theoretically analyzed the fibre laser with two claddings (Figure 19). One of these claddings (the inner one) is composed of glass with $\mathrm{Yb}^{3+}: \mathrm{YLF}$ nanocrystals. It covers the $\mathrm{Yb}^{3+}$-doped fibre glass core. The outer cladding is made of the same glass as the inner cladding, but without $\mathrm{Yb}^{3+}$ :YLF nanocrystals. The authors of this paper expect that the optically pumped $\mathrm{Yb}^{3+}$ :YLF nanocrystals in the inner cladding will lower the local temperature through anti-Stokes fluorescence. They proposed to reduce Rayleigh scattering by index matching the composite host material with the index of YLF. Their model shows a significant temperature decrease of $19 \mathrm{~K}$ when the $\mathrm{Yb}^{3+}: \mathrm{YLF}$ nanocrystals within the composite cladding have a volume fraction of $10 \%$. 


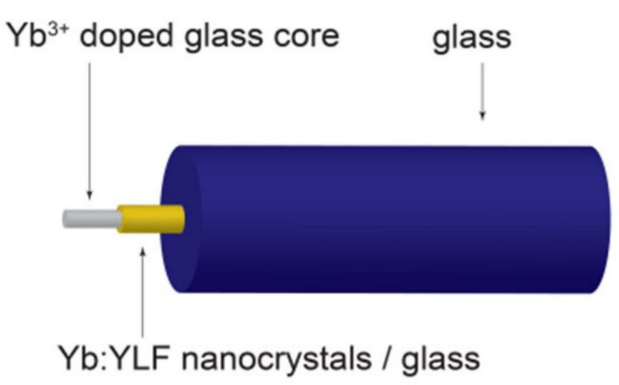

(a)

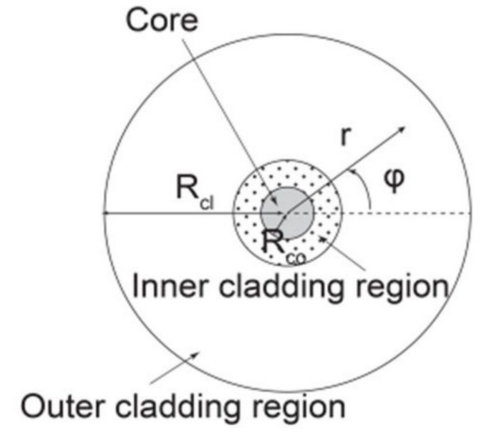

(b)

Figure 19. (a) The core of the fibre is $\mathrm{Yb}^{3+}$-doped glass. The inner region of the cladding (yellow) is composed of glass and YLF nanocrystals, and the outer region of the cladding (blue) is made of the same glass as the inner cladding region, but with no $\mathrm{Yb}^{3+}: \mathrm{YLF}$. (b) The fibre cross section [60].

\subsection{Athermal Spherical Microlasers}

Microlasers fabricated from upconverting nanoparticles (UCNP) coupled to whispering gallery mode (WGM) microresonators were demonstrated in [61,62]. They can exhibit continuous wave anti-Stokes lasing useful for tracking cells, environmental sensing, and coherent stimulation of biological activity. Continuous-wave anti-Stokes lasing with laser thresholds as low as $1.7 \pm 0.7 \mathrm{~kW} / \mathrm{cm}^{2}$ was achieved.

The radiation-balanced version of the spherical microlaser was proposed in [63]. As one can see in Figure 20a, in this athermal microlaser the monolayer of UCNPs is deposited on the surface of the microsphere. This monolayer consists of two different UCNPs: $\mathrm{Yb}^{3+}$-doped $\beta$-NaYF4 UCNPs, which are responsible for optical refrigeration, and $\mathrm{Yb}^{3+} / \mathrm{Er}^{3+} / \mathrm{Tm}^{3+}$-codoped $\beta$-NaYF4 UCNPs, which serve as solid-state gain media. In this scheme, the cooling power and the stimulated emission are provided by UCNPs with different compositions operating at the same pump wavelength.

(a)

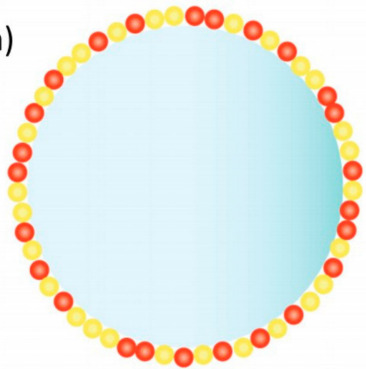

$\mathrm{PS} / \mathrm{SiO}_{2}$ Laser-refrigeration NP, $\mathrm{NaYF}_{4}: \mathrm{Yb}$

(b)

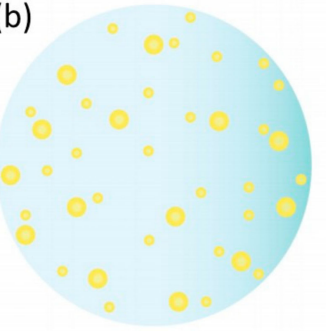

Gain NP, $\mathrm{NaYF}_{4}: \mathrm{Yb} / \mathrm{Er} / \mathrm{Tm}$

Figure 20. Schematics of (a) a radiation-balanced microlaser and (b) a 3D composite laser refrigeration microsphere [63].

The authors of [63] also proposed to use $\mathrm{Yb}^{3+}$-doped UCNPs distributed throughout the entire volume of the microsphere for its optical cooling (Figure 20b). The athermal operation of microsphere lasers has not been demonstrated yet.

\section{Conclusions}

The presented work gives an overview of the latest achievements in a very promising and intensively developing area of laser physics known as radiation-balanced or athermal lasers. It is shown that the radiation balance can be maintained using optical refrigeration based on anti-Stokes fluorescence in the system of RE ions doped in a low-phonon host material. Optical refrigeration is the only solid-state technology that can cool to cryogenic 
temperatures. A basic model describing the operation of radiation-balanced lasers has been presented and discussed. The analytic expressions describing radiation-balanced laser operation were obtained using the set of coupled rate equations. The requirements for radiation balance predict that the characteristics of a radiation-balanced laser are affected by cavity parameters and fundamental material properties. The pump wavelength is the most sensitive parameter. To attain optical refrigeration in the laser, the pump wavelength must exceed the mean fluorescence wavelength.

At the present time, athermal lasers follow four main designs: radiation-balanced bulk and fibre lasers, radiation-balanced disk lasers, and athermal microlasers. All four designs considered in this review are very promising for different applications. Anti-Stokes cooling of RE-doped bulk, fibre, and disk lasers have been experimentally demonstrated. Both experiments and calculations indicate that improving the purity of host crystals will result into improving athermal laser performance. The development of new RE-doped low-phonon materials can facilitate the realization of athermal lasers and accelerate their commercialisation. Recently, Nakayama et al. [64,65] proposed to use $\mathrm{Yb}^{3+}$-doped yttrium aluminum perovskite (YAP) as an active medium of radiation-balanced lasers. YAP has properties that are similar to those of YAG, but it possesses a phonon energy $\left(620 \mathrm{~cm}^{-1}\right)$ that is lower than that of YAG $\left(865 \mathrm{~cm}^{-1}\right)$. This lower phonon energy is a source of higher quantum efficiency. It has been shown that the small-signal gain of athermal laser materials utilizing an $f-f$ transition of RE ions can be controlled by engineering the surrounding crystal fields of the RE dopant.

The further average power scaling requires better control of the beam intensity profile. Recently, athermal laser operation with mode-mismatched Gaussian and super-Gaussian beams was analyzed by Sheik-Bahae and Yang [66]. As one can see in [66], in a disk geometry, beam-area scaling to high-power operations can be accompanied by large transverse temperature gradients. These undesirable gradients can be countered by pump beam shaping and/or employing longer gain media.

Considering four main designs of radiation-balanced lasers, I believe that athermal disk lasers will be the first commercially available radiation-balanced lasers in the coming years. Indeed, the disk shape of a gain medium provides significant freedom in the control of pump power distribution, which is a key element for athermal operation of the laser.

Funding: This research received no external funding.

Institutional Review Board Statement: Not applicable.

Informed Consent Statement: Not applicable.

Data Availability Statement: Not applicable.

Acknowledgments: Not applicable.

Conflicts of Interest: The author declares no conflict of interest.

\section{References}

1. Keyes, R.J.; Quist, T.M. Injection luminescent pumping of CaF2: $\mathrm{U}^{3+}$ with GaAs diode lasers. Appl. Phys. Lett. 1964, 4, 50-52. [CrossRef]

2. Streifer, W.; Scifres, D.; Harnagel, G.; Welch, D.; Berger, J.; Sakamoto, M. Advances in laser diode pumps. IEEE J. Quantum Electron. 1988, 24, 883-894. [CrossRef]

3. Snitzer, E. Proposed fiber cavities for optical masers. Appl. Phys. 1964, 32, 36-39. [CrossRef]

4. Hegarty, J.; Broer, M.M.; Golding, B.; Simpson, J.R.; MacChesney, J.B. Photon echoes below $1 \mathrm{~K}$ in a Nd $\mathrm{Nd}^{3+}$ - doped glass fiber. Phys. Rev. Lett. 1983, 51, 2033-2035. [CrossRef]

5. Poole, S.B.; Payne, D.N.; Fermann, M.E. Fabrication of low-loss optical fibers contalning rare-earth ions. Electron. Lett. 1985, 21, 737-738. [CrossRef]

6. Poole, S.B.; Payne, D.N.; Mears, R.J.; Fermann, M.E.; Laming, R.L. Fabrication and characterization of low loss optical fibers containing rare earth ions. J. Lightwave Tech. 1986, LT-4, 870-876. [CrossRef]

7. Mears, R.J.; Reekie, L.; Poole, S.B.; Payne, D.N. Neodymium-doped silica single-miber lasers. Electron. Lett. 1985, $21,737-738$. [CrossRef] 
8. Taverner, D.; Richardson, D.J.; Dong, L.; Caplen, J.E.; Williams, K.; Penty, R.V. 158-pJ pulses from a singletransverse-mode, large-mode-area erbium-doped fiber amplifier. Opt. Lett. 1997, 22, 378-380. [CrossRef]

9. Nemova, G.; Kashyap, R. High-power long period grating assisted EDFA. J. Opt. Soc. Am. B 2008, 25, 1322-1327. [CrossRef]

10. Martin, W.S.; Chernoch, J.P. Multiple Internal Reflection Face-Pumped Laser. U.S. Patent 3633126A, 4 January 1972.

11. Eggleston, J.M.; Frantz, L.M.; Injeyan, H. Derivation of the Frantz-Nodvik equation for zig-zag optical path, slab geometry laser amplifiers. IEEE J. Qunntum Electron. 1989, 25, 1855-1862. [CrossRef]

12. Giesen, A.; Hiigel, H.; Voss, A.; Wittig, K.; Brauch, U.; Opower, H. Scalable concept for diode-pumped high-power solid-state lasers. Appl. Phys. B 1994, 58, 365-372. [CrossRef]

13. Giesen, A.; Hugel, H.; Voss, A.; Wittig, K.; Brauch, U.; Opower, H. Diocle-Pumped high-power solid-state laser: Concept and first results with Yb:YAG. In Advanced Solid State Lasers; OSA Proceeding Series; Optical Society of America: Washington, DC, USA, 1994.

14. Bowman, S.R. Laser without internal heat generation. IEEE J. Quantum Electron. 1999, 35, 115-122. [CrossRef]

15. Nemova, G.; Kashyap, R. Laser cooling of solids. Rep. Prog. Phys. 2010, 73, 086501. [CrossRef]

16. Koechner, W. Absorbed pump power, thermal profile and stresses in a cw pumped Nd: YAG crystal. Appl. Opt. 1970, 9, 1429-1434. [CrossRef]

17. Schmid, M.; Graf, T.; Weber, H.P. Analytical model of the temperature distribution and the thermally induced birefringence in laser rods with cylindrically symmetric heating. J. Opt. Soc. Am. B 2000, 17, 1398-1404. [CrossRef]

18. Farrukh, U.; Buoncristiani, A.; Byvik, C. An analysis of the temperature distribution in finite solid-state laser rods. IEEE J. Quantum Electron. 1988, 34, 2253-2263. [CrossRef]

19. Innocenzi, M.; Yura, H.; Fincher, C.; Fields, R. Thermal modeling of continuous-wave end-pumped solidstate lasers. Appl. Phys. Lett. 1990, 56, 1831-1833. [CrossRef]

20. Chen, Y.; Huang, T.; Kao, C.; Wang, C.; Wang, S. Optimization in scaling fiber-coupled laser-diode endpumped lasers to higher power: Influence on thermal effect. IEEE J. Quantum Electron. 1997, 33, 1424-1429. [CrossRef]

21. Cousins, A. Temperature and thermal stress scaling in finite-length end-pumped laser rods. IEEE J. Quantum Electron. 1992, 28, 1057-1069. [CrossRef]

22. Pringsheim, P. Zwei Bemerkungen über den Unterschied von Lumineszenz- und Temperaturstrahlung. Z. Phys. 1929, 57, 739-746. [CrossRef]

23. Epstein, R.I.; Buchwald, M.I.; Edwards, B.C.; Gosnell, T.R.; Mungan, C.E. Observation of laser-induced fluorescent cooling of a solid. Nat. Cell Biol. 1995, 377, 500-502. [CrossRef]

24. Sheik-Bahae, M.; Epstein, R.I. Optical refrigeration. Nat. Photonics 2007, 1, 693-699. [CrossRef]

25. Andrianov, S.N.; Samartsev, V.V. Solid-state lasers with internal laser refrigeration effect. In Proceedings of the SPIE PECS'2001: Photon Echo and Coherent Spectroscopy, Nizhny Novgorod, Russia, 15 November 2001; Volume 4605, pp. $208-213$.

26. Nemova, G.; Kashyap, R. Athermal continuous-wave fiber amplifier. Opt. Commun. 2009, 282, 2571-2575. [CrossRef]

27. Auzel, F. Upconversion and anti-Stokes processes with $\mathrm{f}$ and d ions in solids. Chem. Rev. 2004, 104, 139-173. [CrossRef]

28. Mungan, C.E. Thermodynamics of radiation-balanced lasing. J. Opt. Soc. Am. B 2003, 20, 1075-1082. [CrossRef]

29. Bowman, S.R.; Jenkins, N.W.; O'Connor, S.P.; Feldman, B.J. Sensitivity of stability of a radiation-balanced laser system. IEEE J. Quantum Electron. 2002, 38, 1339-1348. [CrossRef]

30. Bowman, S.R.; Mungan, C. New materials for optical cooling. Appl. Phys. B 2000, 71, 807-811. [CrossRef]

31. Bowman, S.R.; Jenkins, N.W.; Feldman, B.; O'Connor, S. Demonstration of a radiatively cooled laser. In Proceedings of the Conference on Lasers and Electro-Optics (CLEO), Long Beach, CA, USA, 24 May 2002.

32. Bowman, S.R.; O'Connor, S.; Biswal, S. Ytterbium laser with reduced thermal heating. IEEE J. Quantum Electron. 2005, 41, 1510-1517. [CrossRef]

33. Bowman, S.R.; O'Connor, S.P.; Biswal, S.; Condon, N.J.; Rosenberg, A. Minimizing heat generation in solid-state lasers. IEEE J. Quantum Electron. 2010, 46, 1076-1085. [CrossRef]

34. Bowman, S.R. Optically cooled lasers. In Laser Cooling: Fundamental Properties and Application; Nemova, G., Ed.; Pan Stanford Publishing Pte. Ltd.: Singapore, 2016.

35. Nemova, G.; Kashyap, R. Thin-disk athermal laser system. Opt. Commun. 2014, 319, 100-105. [CrossRef]

36. Yang, Z.; Meng, J.; Albrecht, A.R.; Sheik-Bahae, M. Radiation-balanced Yb:YAG disk laser. Opt. Express 2019, 27, 1392-1400. [CrossRef]

37. Yang, Z.; Meng, J.; Albrecht, A.R.; Kock, J.; Sheik-Bahae, M. Radiation-balanced thin-disk lasers in Yb:YAG and Yb:YLF. In Proceedings of the SPIE Photonic Heat Engines: Science and Applications, San Francisco, CA, USA, 2-7 February 2019 ; p. 23.

38. Volpi, A.; Kocka, J.; Albrechta, A.R.; Rostamia, S.; Hehlenb, M.P.; Sheik-Bahae, M. Mode scaling in radiation balanced disk lasers for various gain materials. In Proceedings of the SPIE Photonic Heat Engines: Science and Applications III, Online, 6-12 March 2021; Volume 11702, p. 117020U.

39. Nemova, G.; Kashyap, R. Fiber amplifier with integrated optical cooler. J. Opt. Soc. Am. B 2009, 26, 2237-2241. [CrossRef]

40. Nemova, G.; Kashyap, R. Raman fiber amplifier with integrated cooler. IEEE J. Light. Technol. 2009, 27, 5597-5601. [CrossRef]

41. Nemova, G.; Kashyap, R. High-power fiber lasers with integrated rare-earth optical cooler. In Proceedings of the SPIE Laser Refrigeration of Solids III, San Francisco, CA, USA, 23-28 January 2010; Volume 7614, pp. 761406-761416. 
42. Mafi, A. Temperature distribution inside a double-cladding optical fiber laser or amplifier. J. Opt. Soc. Am. B 2020, 37, 1821-1828. [CrossRef]

43. Peysokhan, M.; Mobini, E.; Allahverdi, A.; Abaie, B.; Mafi, A. Characterization of Yb-doped ZBLAN fiber as a platform for radiation-balanced lasers. Photonics Res. 2020, 8, 202-210. [CrossRef]

44. Peysokhan, M.; Mobini, E.; Mafi, A. Analytical formulation of a high-power Yb-doped double-cladding fiber laser. OSA Contin. 2020, 3, 1940-1951. [CrossRef]

45. Vigneron, P.B.; Knalla, J.; Boilardb, T.; Bernierb, M.; Digonnet, M.J.F. Observation of anti-Stokes-fluorescence cooling in a ZBLAN fiber with a Yb-doped cladding. In Proceedings of the SPIE OPTO, Online, 5 April 2021; Volume 11702, p. 117020A.

46. Gosnell, T.R. Laser cooling of a solid by $65 \mathrm{~K}$ starting from room temperature. Opt. Lett. 1999, 24, 1041-1043. [CrossRef]

47. Knall, J.M.; Arora, A.; Bernier, M.; Digonnet, M.J.F. Anti-stokes fluorescence cooling in Yb-doped ZBLAN fibers at atmospheric pressure: Experiments and near-future prospects. In Proceedings of the SPIE Photonic Heat Engines: Science and Applications, San Francisco, CA, USA, 2-7 February 2019; Volume 10936, p. 109360F.

48. Knall, J.; Arora, A.; Bernier, M.; Cozic, S.; Digonnet, M.J.F. Demonstration of anti-Stokes cooling in Ybdoped ZBLAN fibers at atmospheric pressure. Opt. Lett. 2019, 44, 2338-2341. [CrossRef] [PubMed]

49. Arora, A.; Esmaeelpour, M.; Bernier, M.; Digonnet, M.J.F. High-resolution slow-light fiber Bragg grating temperature sensor with phase-sensitive detection. Opt. Lett. 2018, 43, 3337-3340. [CrossRef] [PubMed]

50. Ytterbium Single-Mode CW Systems. Available online: http://www.ipgphotonics.com/en/products/lasers/high-powercwfiber-lasers/1-micron/yls-sm-1-10-kw (accessed on 13 August 2021).

51. Aydin, Y.O.; Fortin, V.; Vallée, R.; Bernier, M. Towards power scaling of $2.8 \mu \mathrm{m}$ fiber lasers. Opt. Lett. 2018, $43,4542-4545$. [CrossRef]

52. Knall, J.; Vigneron, P.-B.; Engholm, M.; Dragic, P.D.; Yu, N.; Ballato, J.; Bernier, M.; Digonnet, M.J.F. Laser cooling in a silica optical fiber at atmospheric pressure. Opt. Lett. 2020, 45, 1092-1095. [CrossRef]

53. Mobini, E.; Rostami, S.; Peysokhan, M.; Albrecht, A.; Kuhn, S.; Hein, S.; Hupel, C.; Nold, J.; Haarlammert, N.; Schreiber, T.; et al. Laser cooling in silica glass. arXiv 2020, arXiv:1910.10609v1.

54. Knall, J.; Engholm, M.; Ballato, J.; Dragic, P.D.; Yu, N.; Digonnet, M.J.F. Experimental comparison of silica fibers for laser cooling. Opt. Lett. 2002, 45, 4020-4023. [CrossRef] [PubMed]

55. Knall, J.M.; Engholm, M.; Boilard, T.; Bernier, M.; Digonnet, M.J.F. A radiation-balanced silica fiber amplifier. arXiv 2021, arXiv:2103.02698.

56. Knall, J.M.; Digonnet, M.J.F. Design of high-power radiation-balanced silica fiber lasers with a doped core and cladding. J. Light. Technol. 2021, 39, 2497-2504. [CrossRef]

57. Nemova, G.; Kashyap, R. Laser cooling with $\mathrm{Tm}^{3+}$-doped nano-crystals of oxy-fluoride glass ceramic. In Proceedings of the SPIE Photonics Europe, Brussels, Belgium, 1 May 2012; Volume 8424, p. 84242I.

58. Nemova, G.; Kashyap, R. Laser cooling with $\mathrm{Tm}^{3+}$-doped oxy-fluoride glass ceramic. J. Opt. Soc. Am. B 2012, 29, 3034-3038 [CrossRef]

59. Xia, X.; Pauzauskie, P.J.; Pant, A.; Davis, E.J. Laser refrigeration of optical fibers via optically-active composite cladding materials. In Proceedings of the SPIE Photonic Heat Engines: Science and Applications, San Francisco, CA, USA, 2-7 February 2019; Volume 10936, p. 109360I.

60. Xia, X.; Pant, A.; Davis, E.J.; Pauzauskie, P.J. Design of a radiation-balanced fiber-laser via optically active composite cladding materials. J. Opt. Soc. Am. B 2019, 36, 3307-3314. [CrossRef]

61. Fernandez-Bravo, A.; Yao, K.; Barnard, E.S.; Borys, N.J.; Levy, E.S.; Tian, B.; Tajon, C.A.; Moretti, L.; Altoe, M.V.; Aloni, S.; et al. Continuous-wave upconverting nanoparticle microlasers. Nat. Nanotechnol. 2018, 13, 572-577. [CrossRef]

62. Liu, Y.; Teitelboim, A.; Fernandez-Bravo, A.; Yao, K.; Altoe, M.V.P.; Aloni, S.; Zhang, C.; Cohen, B.E.; Schuck, P.J.; Chan, E.M. Controlled Assembly of Upconverting Nanoparticles for Low-Threshold Microlasers and Their Imaging in Scattering Media. ACS Nano 2020, 14, 1508-1519. [CrossRef]

63. Xiaa, X.; Pantb, A.; Felstedb, R.G.; Gariepyb, R.E.; Davisc, E.J.; Pauzauskie, P.J. Radiation balanced spherical microlaser. In Proceedings of the SPIE Photonic Heat Engines: Science and Applications III, Online, 6-12 March 2021; Volume 11702, p. 117020R.

64. Nakayama, Y.; Harada, Y.; Kita, T. An energy transfer accompanied by phonon absorption in ytterbium-doped yttrium aluminum perovskite for optical refrigeration. Appl. Phys. Lett. 2020, 117, 041104. [CrossRef]

65. Nakayama, Y.; Harada, Y.; Kita, T. Yb-doped yttrium aluminum perovskite for radiation balanced laser application. In Proceedings of the SPIE Photonic Heat Engines: Science and Applications III, Online, 6-12 March 2021; Volume 11702, p. 117020K.

66. Sheik-Bahae, M.; Yang, Z. Optimum Operation of Radiation-Balanced Lasers. IEEE J. Quantum Electron. 2020, 56, 1000109. [CrossRef] 